\title{
Theoretical investigation of mixing in warm clouds - Part 2: Homogeneous mixing
}

\author{
Mark Pinsky $^{1}$, Alexander Khain ${ }^{1}$, Alexei Korolev ${ }^{2}$, and Leehi Magaritz-Ronen ${ }^{1}$ \\ ${ }^{1}$ Department of Atmospheric Sciences, The Hebrew University of Jerusalem, Jerusalem, Israel \\ ${ }^{2}$ Environment Canada, Cloud Physics and Severe Weather Section, Toronto, Canada \\ Correspondence to: Alexander Khain (alexander.khain@mail.huji.ac.il)
}

Received: 26 August 2015 - Published in Atmos. Chem. Phys. Discuss.: 4 November 2015

Revised: 30 May 2016 - Accepted: 3 June 2016 - Published: 28 July 2016

\begin{abstract}
Evolution of monodisperse and polydisperse droplet size distributions (DSD) during homogeneous mixing is analyzed. Time-dependent universal analytical expressions for supersaturation and liquid water content are derived. For an initial monodisperse DSD, these quantities are shown to depend on a sole non-dimensional parameter. The evolution of moments and moment-related functions in the course of homogeneous evaporation of polydisperse DSD is analyzed using a parcel model.

It is shown that the classic conceptual scheme, according to which homogeneous mixing leads to a decrease in droplet mass at constant droplet concentration, is valid only in cases of monodisperse or initially very narrow polydisperse DSD. In cases of wide polydisperse DSD, mixing and successive evaporation lead to a decrease of both mass and concentration, so the characteristic droplet sizes remain nearly constant. As this feature is typically associated with inhomogeneous mixing, we conclude that in cases of an initially wide DSD at cloud top, homogeneous mixing is nearly indistinguishable from inhomogeneous mixing.
\end{abstract}

\section{Introduction}

Turbulent mixing at cloud edges and cloud tops accompanied by phase transitions has been the focus of numerous studies, beginning with the pioneering works of Baker and Latham (1979), Baker et al. (1980), Blyth et al. (1980) and Baker and Latham (1982). Laboratory experiments by Latham and Reed (1977) showed that after mixing with subsaturated air some droplets completely evaporate while others remain unchanged. This finding gave rise to the concept of two types of turbulent mixing: homogeneous and inhomogeneous. A recent description of the classical concepts of homogeneous and inhomogeneous mixing can be found in study by Korolev et al. (2016), hereafter referred to as Pt1.

Figure 1 presents a conceptual scheme of homogeneous mixing between saturated cloud volume $V_{1}$ containing droplets and sub-saturated droplet-free volume $V_{2}$ (Fig. 1a) in case of initially monodisperse droplet size distribution (DSD). This scheme is the base of further analysis. According to the concept of homogeneous mixing, the air within the volumes mixes at a rate much higher than the characteristic rate of droplet evaporation. So, the fields of temperature and humidity (and, therefore, the fields of the relative humidity and supersaturation) are rapidly homogenized throughout the entire volume, and all the droplets experience the same supersaturation (Fig. 1b). At the end of the first stage, the droplet concentration decreases due to dilution down to $N_{\mathrm{m} 0}=N_{1} \frac{V_{1}}{V_{1}+V_{2}}$, where $N_{1}$ is the initial droplet concentration in the cloud volume. At the second stage (Fig. 1c), droplets change supersaturation and temperature through their evaporation. There are two possible scenarios for the final equilibrium states. In the first one, illustrated in Fig. 1c, droplets continue evaporating until they saturate the environment. The size of all droplets decreases, but they evaporate only partially, so the droplet concentration $N_{\mathrm{m} 0}$ remains unchanged. In the second scenario, when the initially droplet-free volume is very dry, the droplets penetrated from cloud volume evaporate completely. In case of polydisperse initial DSD, both partial and complete evaporation of droplets determine the final DSD.

In contrast to homogeneous mixing, spatial homogenization during inhomogeneous mixing is a relatively slow pro- 


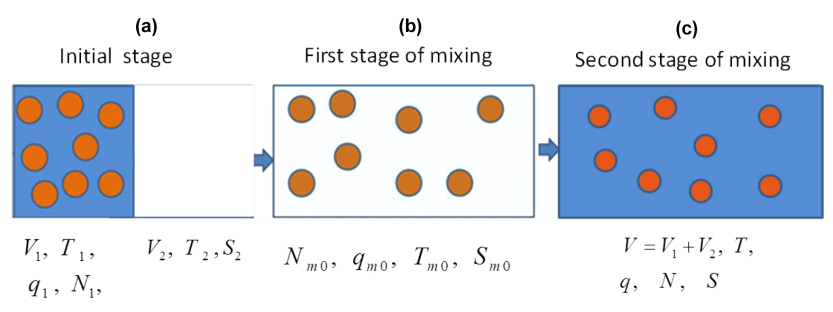

Figure 1. Conceptual scheme of homogeneous mixing in case of monodisperse DSD. The subsaturated volume of dry air is colored white, and the cloudy volumes with saturated air are colored dark blue. The volume forming as a result of mixing after total homogenization is colored light blue. Index 1 shows the initial values characterizing the initially cloudy volume. Index 2 denotes initial values in the droplet-free volume. Index "m0" denotes values in the mixing volume after the first stage of mixing.

cess. According to the concept of extremely inhomogeneous mixing, some droplets are transported by the turbulent eddies into the dry environment and experience complete evaporation, whereas other droplets remain unchanged. As in the case of homogeneous mixing, the process of droplet evaporation continues until either the environment is saturated or all the droplets evaporate. According to the classical concept, during extremely inhomogeneous mixing the shape of DSD is conserved, however, the total droplet concentration decreases (see review by Devenish et al., 2012, and Pt1).

The classical concepts analyze only the final equilibrium states which are based on the mass conservation consideration. Strictly speaking, the size distributions in the final states, assumed in the classical concepts, are hypothetical and cannot be reached. This is because the classic concepts do not take into account the mixing-induced DSD broadening. Detailed simulation of time evolution of DSD and other microphysical parameters is necessary not only to better determine the final states, but also to evaluate time periods during which such final states are reached. The analysis of time evolution is practically important because many DSDs measured in situ correspond to the transient state, but not to the final equilibrium state. Besides, it is necessary to determine the evolution of initially polydisperse DSD, that may substantially differ from the evolution of monodisperse DSD.

We analyze the time evolution of the DSD during homogeneous mixing in cases of monodisperse and polydisperse initial DSDs. The analysis is based on new equations and methodology developed by Pinsky et al. (2013, 2014).

First, we need to evaluate conditions at which mixing can be considered homogeneous. The characteristic spatial scale of homogeneous mixing can be estimated by comparing the characteristic times of two processes providing thermodynamic equilibrium inside a mixing volume. The first process is mechanical mixing (diffusion) governed by turbulence. Turbulent mixing leads to homogenization of temperature, humidity (and, thus, of supersaturation) as well as of droplet concentration within the volume $V=V_{1}+V_{2}$. The second process is evaporation of a droplet ensemble, which leads to an increase in relative humidity and to the thermodynamical equilibrium in the mixing volume.

The process of mixing that is accompanied by droplet evaporation is characterized by two timescales. The first timescale is the characteristic mixing (homogenization) time $\tau_{\text {mix }}$ of an entrained volume with linear scale $L_{\text {mix }}$ can be evaluated from the relationship (Monin and Yaglom, 1975)

$\tau_{\operatorname{mix}}=\varepsilon^{-1 / 3} L_{\operatorname{mix}}^{2 / 3}$,

where $\varepsilon$ is the turbulent kinetic energy dissipation rate. Equation (1) suggests that the size of the volume falls within the inertial interval of turbulence. Therefore, after the time $\tau_{\text {mix }}$, volume with a linear scale of about $L_{\text {mix }}$ will be mechanically homogenized and all the droplets in the volume will experience the same supersaturation.

The second timescale characterizes rate of droplet evaporation and corresponding changes of supersaturation. In this study, as well as later in study by Pinsky et al. (2016) (hereafter, Part 3), it will be shown that the characteristic evaporation time is the phase relaxation time $\tau_{\mathrm{pr}}$, which determines the rate of change of supersaturation during condensation or evaporation (Mazin, 1968; Korolev and Mazin, 2003)

$\tau_{\mathrm{pr}}=(4 \pi D \bar{r} N)^{-1}$,

where $N=N_{\mathrm{m} 0}$ is the concentration of droplets in the mixing volume, $\bar{r}$ is the mean radius of droplets and $D$ is the diffusivity of water vapor. The spatial scale at which the mixing time is equal to the phase relaxation time is called a phase relaxation scale $L_{\text {pr }}$ (Mazin, 1968). This scale can be calculated from Eqs. (1) and (2) as

$L_{\mathrm{pr}}=\varepsilon^{1 / 2} \tau_{\mathrm{pr}}^{3 / 2} \approx \varepsilon^{1 / 2}(4 \pi D \bar{r} N)^{-3 / 2}$.

The type of mixing is often characterized by the value of the Damköhler number which in regard to atmospheric mixing is defined as the ratio $\tau_{\text {mix }} / \tau_{\text {pr }}$ (Baker et al., 1980; Jeffery, 2007; Lehmann et al., 2009):

$D a=\frac{\tau_{\mathrm{mix}}}{\tau_{\mathrm{pr}}}=\frac{4 \pi D \bar{r} N L_{\mathrm{mix}}^{2 / 3}}{\varepsilon^{1 / 3}}$.

The case $D a \ll 1$ corresponds to homogeneous mixing, when mechanical homogenization occurs much faster than droplet evaporation. The case $D a \gg 1$ corresponds to extremely inhomogeneous mixing. It is reasonable to consider the value $D a=1$ as a boundary separating the two types of mixing. This condition is equivalent to the condition

$L_{\text {mix }}=L_{\mathrm{pr}} \approx \varepsilon^{1 / 2}(4 \pi D \bar{r} N)^{-3 / 2}$.

Expression (5) determines the maximum spatial scale at which mixing can be considered as homogeneous. The evaluation of the spatial scales at conditions typical of different 
Table 1. Linear scales of volumes experiencing homogeneous mixing at conditions typical of different cloud types.

\begin{tabular}{lrrrrrr}
\hline Cloud type & $N, \mathrm{~cm}^{-3}$ & $\mathrm{LWC}_{\mathrm{g} \mathrm{m}}{ }^{-3}$ & $r, \mu \mathrm{m}$ & Dissipation rate, $\mathrm{cm}^{2} \mathrm{~s}^{-3}$ & Phase relaxation time, $\mathrm{s}$ & Phase scale, $\mathrm{m}$ \\
\hline Maritime convective & 100 & 2.0 & 16.8 & 300 & 2.01 & 0.49 \\
Maritime stratocumulus & 100 & 0.5 & 10.6 & 10 & 3.19 & 0.18 \\
Weak stratocumulus & 100 & 0.2 & 7.8 & 5 & 4.33 & 0.2 \\
Continental convective & 500 & 2 & 8.0 & 500 & 0.75 & 0.6 \\
\hline
\end{tabular}

cloud types is presented in Table 1 . One can see that the characteristic volume size at which mixing can be considered homogeneous ranges from 0.2 to $0.6 \mathrm{~m}$. At larger scales, supersaturation within the mixing volume is non-uniform and droplets in the volume experience different values of relative humidity. In this case, the mixing should be considered inhomogeneous.

The structure of the paper is as follows. In Sect. 2 we calculate the thermodynamic characteristics of the resulting volume at the end of the first stage of mixing (see Fig. 1b). Section 3 presents an analytic solution of homogeneous droplet evaporation in the monodisperse DSD case. In Sect. 4 effects of polydispersivity on the DSD evolution are described. The problem of turbulent mixing representation in numerical cloud models is discussed in Sect. 5. The main results of the study are presented in Sect. 6 .

\section{Thermodynamic characteristics of the mixing volume at the end of the first stage}

At the first stage (see the scheme in Fig. 1) homogeneous mixing is considered as an isobaric process that is not accompanied by phase transitions (Korolev and Isaac, 2000). Let us consider mixing between a cloud volume with mass $m_{1}$, supersaturation $S_{1}=0$ and temperature $T_{1}$ and a dropletfree volume with mass $m_{2}$, supersaturation $S_{2}<0$ and temperature $T_{2}$. The cloud volume also contains droplets with concentration $N_{1}$ and liquid water mixing ratio $q_{1}$ (Fig. 1). For the sake of simplicity, we assume that the mass of both volumes is equal to one. Let us further assume that $\mu$ is the mass fraction of the cloud air that mixes with the mass fraction $(1-\mu)$ of the droplet-free air. In this case, the air mass in the mixing volume will be equal to $m_{1} \mu+(1-\mu) m_{2}=1$. Isobaric mixing leads to an approximate linear dependence of droplet concentration $N_{\mathrm{m} 0}$ on $\mu$.

After an instantaneous homogenization of the two volumes, the intermediate temperature $T_{\mathrm{m} 0}$, droplet concentration $N_{\mathrm{m} 0}$ and liquid mixing ratio $q_{\mathrm{m} 0}$ are (see Pt1):

$N_{\mathrm{m} 0}=N_{1} \mu ; \quad q_{\mathrm{m} 0}=q_{1} \mu ; \quad T_{0}=T_{1} \mu+T_{2}(1-\mu)$.

If the temperature difference $\left|T_{1}-T_{2}\right|$ does not exceed a few degrees, the intermediate supersaturation $S_{\mathrm{m} 0}$ can be approximated by a linear dependence on $\mu$

$S_{\mathrm{m} 0}=S_{2}(1-\mu)$.

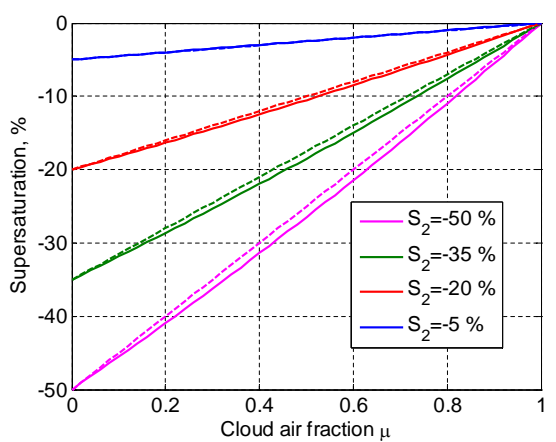

Figure 2. Dependence of supersaturation on parameter $\mu$ : simulation results (solid line) and an approximate linear dependence calculated using Eq. (6b) (dashed line). The initial temperatures of two volumes are $T_{1}=8^{\circ} \mathrm{C}$ and $T_{2}=10^{\circ} \mathrm{C}$.

Figure 2 shows that in case $\left|T_{1}-T_{2}\right|<2{ }^{\circ} \mathrm{C}$, the deviations of supersaturation from the linear dependence (Eq. 6b) are small enough and can be neglected. In cases when the temperature of the dry volume substantially differs from the temperature of the cloud volume, the dependence of the resulting supersaturation on parameter $\mu$ becomes non-linear (Fig. 2). At temperature differences of $5-10^{\circ} \mathrm{C}$, the deviation from the analytical solution (6) increases, which requires using more precise formulas for supersaturation (see Pt1). Values of $N_{\mathrm{m} 0}, q_{\mathrm{m} 0}, T_{\mathrm{m} 0}$ and $S_{\mathrm{m} 0}$ determine the initial conditions for the second stage of homogeneous mixing. This is actually homogeneous evaporation of droplets, which leads to a thermodynamic equilibrium between water vapor and liquid water.

\section{Analysis of homogeneous droplet evaporation in the monodisperse DSD case}

\subsection{Basic assumptions and equations}

The second stage of mixing consists of homogeneous evaporation of droplets. The evolution of DSD during the second stage is considered here under the following assumptions: (a) the processes inside the mixing volume are adiabatic, (b) the droplet size distribution is monodisperse, (c) the vertical velocity of the volume $u_{z}=0$ and (d) the sedimentation of droplets is neglected and their concentration remains constant. 
The liquid water mixing ratio can be expressed as

$q(t)=\frac{4 \pi \rho_{\mathrm{w}}}{3 \rho_{\mathrm{a}}} N r^{3}(t)$,

where $r(t)$ is the radius of droplets and $N$ is the droplet number concentration. Closed equations describing condensation and/or evaporation in a moving adiabatic air volume were obtained by Pinsky et al. (2013). In an unmoving adiabatic volume, evaporation is described by the equation for supersaturation (e.g., Korolev and Mazin, 2003)

$\frac{1}{S+1} \frac{\mathrm{d} S}{\mathrm{~d} t}=-A_{2} \frac{\mathrm{d} q}{\mathrm{~d} t}$

and droplet evaporation is described by the simplified equation (Pruppacher and Klett, 1997)

$r \frac{\mathrm{d} r}{\mathrm{~d} t}=\frac{S}{F}$,

where $S$ is the supersaturation over a flat water surface. For cloud droplets Eq. (9) is valid with high accuracy. We do not take into account formation of haze particles resulting from droplet evaporation. This allows us to neglect the curvature term and chemical term in the evaporation equation. Coefficients $A_{2}$ and $F$ in Eqs. (8) and (9) are slightly dependent on the temperature and the pressure

$A_{2}=\frac{1}{q_{\mathrm{v}}}+\frac{L^{2}}{c_{p} R_{\mathrm{v}} T^{2}}$

$F=\frac{\rho_{\mathrm{W}} L^{2}}{k_{\mathrm{a}} R_{\mathrm{V}} T^{2}}+\frac{\rho_{\mathrm{W}} R_{\mathrm{v}} T}{e_{S}(T) D}$.

We assume that coefficients $A_{2}$ and $F$ do not change in the course of droplet evaporation.

The physical meaning and units of other variables are given in Appendix A.

\subsection{Time evolution of supersaturation and of liquid water content}

The closed differential equations for the liquid water mixing ratio $q$ and supersaturation $S$ to be used in the analysis are derived in Appendix B.

$$
\begin{aligned}
& \frac{\mathrm{d} q}{\mathrm{~d} t}=B N_{\mathrm{m} 0}^{2 / 3}\left[\left(S_{\mathrm{m} 0}+1\right) \exp \left\{-A_{2}\left(q-q_{\mathrm{m} 0}\right)\right\}-1\right] q^{1 / 3} \\
& \frac{1}{S+1} \frac{\mathrm{d} S}{\mathrm{~d} t}=-A_{2} B N_{\mathrm{m} 0}^{2 / 3} S\left(q_{\mathrm{m} 0}-\frac{1}{A_{2}} \ln \frac{S+1}{S_{\mathrm{m} 0}+1}\right)^{1 / 3},
\end{aligned}
$$

where

$B=\frac{3}{F}\left(\frac{4 \pi \rho_{\mathrm{w}}}{3 \rho_{\mathrm{a}}}\right)^{2 / 3}=$ const.

The solutions of these equations depend on the values of $N_{\mathrm{m} 0}, q_{\mathrm{m} 0}, T_{\mathrm{m} 0}$, and $S_{\mathrm{m} 0}$, obtained after the first stage of mixing. Equations (12) and (13) are rigidly connected by the following equation directly following from Eq. (8):

$\ln [S(t)+1]=-A_{2} q(t)+C$, where $C=\ln \left[S_{\mathrm{m} 0}+1\right]+A_{2} q_{\mathrm{m} 0}$ is determined by the initial conditions at $t=0$.

Since in this study we assume that $S(t) \leq 0$, it is convenient to use the relative humidity $\mathrm{RH}$ and the saturation deficit SD to characterize the thermodynamic state of the mixing volume. Both quantities are easily related to $S(t)$ : $\mathrm{SD}(t)=-S(t), \mathrm{RH}(t)=1+S(t)$. Figure 3 demonstrates dependencies $S(t)$ and $q(t)$, calculated at an initial relative humidity $\mathrm{RH}_{0}$ that varies from 72 to $91.6 \%$, and an initial LWC of $0.6 \mathrm{~g} \mathrm{~m}^{-3} \cdot \mathrm{RH}_{0}$ corresponds to the relative humidity in dry volume $\mathrm{RH}_{2}$, that ranges from 43 to $83 \%$ at $\mu=0.5$. The results of solving Eqs. (14)-(15) were compared with those obtained using a parcel model (Korolev, 1995) in which evaporation is described using equations with temperaturedependent parameters, and were found to be in excellent agreement. This agreement can be attributed to the fact that temperature changes that occurred in the course of the mixing are relatively small, validating the assumption about the constancy of $A_{2}$ and $F$.

As seen from Fig. 3, the final equilibrium state may be reached within several seconds. Figure 3 shows the possibility of the two final states mentioned above: (a) complete droplet evaporation reached at different time instances depending on the initial value of $S_{\mathrm{m} 0}$, and (b) partial droplet evaporation at $\mathrm{RH}_{0}>82 \%$. In the latter case, the final supersaturation is equal to zero.

\subsection{Universal dependencies of supersaturation and of LWC on time}

In order to simplify the further analysis we introduce the following non-dimensional parameters: normalized liquid water mixing ratio $\widetilde{q}=\frac{q}{q_{\mathrm{m} 0}}$ which is equal to normalized liquid water content, normalized supersaturation $\widetilde{S}=$ $\frac{S}{A_{2} q_{\mathrm{m} 0}}$, and non-dimensional time $t=t / \tau_{\mathrm{m} 0}$, where $\tau_{\mathrm{m} 0}=$ $\left(B A_{2} N_{\mathrm{m} 0}^{2 / 3} q_{\mathrm{m} 0}^{1 / 3}\right)^{-1}$ is the timescale. Then the set of nondimensional equations describing changes of supersaturation and liquid mixing ratio can be written as (see Appendix B for detail)

$$
\begin{aligned}
& \widetilde{S}(\widetilde{t})=-\widetilde{q}(\widetilde{t})+\gamma \\
& \frac{\mathrm{d} \widetilde{q}}{\mathrm{~d} \widetilde{t}}=\widetilde{q}^{1 / 3}(\gamma-\widetilde{q}) \\
& \frac{\mathrm{d} \widetilde{S}}{\mathrm{~d} \widetilde{t}}=-(\gamma-\widetilde{S})^{1 / 3} \widetilde{S},
\end{aligned}
$$

where

$\gamma=1+\frac{S_{\mathrm{m} 0}}{A_{2} q_{\mathrm{m} 0}}=1+\frac{1-\mu}{\mu} \frac{S_{2}}{A_{2} q_{1}}$

is a dimensionless parameter which depends on the initial supersaturation $S_{\mathrm{m} 0}$ and the initial liquid water mixing ratio $q_{\mathrm{m} 0}$. The value of this parameter can be either positive or negative. Equations (16)-(18) are strictly valid if $\left|S_{\mathrm{m} 0}\right| \ll 1$, i.e., 

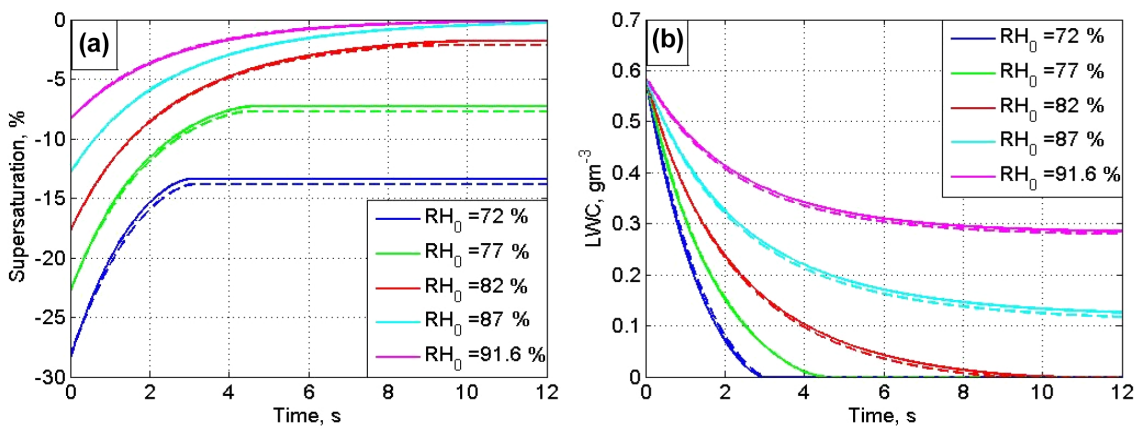

Figure 3. Dependencies $S(t)$ (a) and $q_{\mathrm{w}}(t)(\mathbf{b})$, calculated at different initial relative humidity $\mathrm{RH}_{0}$ using closed differential Eqs. (12) and (13) (solid lines) and using a parcel model (dashed lines). The calculation parameters are $T_{\mathrm{m} 0}=10^{\circ} \mathrm{C}, p_{0}=842 \mathrm{mb}, r_{0}=10 \mu \mathrm{m}$, $N_{\mathrm{m} 0}=140 \mathrm{~cm}^{-3}, q_{\mathrm{w} 0}=0.58 \mathrm{~g} \mathrm{~m}^{-3}$.

when the value of supersaturation $S$ is negligible in comparison with unity in the factor $(S+1)^{-1}$ on the left-hand side of Eq. (8). However, a detailed comparison of solutions of Eqs. (16)-(18) with those obtained using a numerical model showed that Eqs. (16)-(18) provide an accurate solution at $\mathrm{RH}_{0}$ as low as 30-40\%. Equation (17) should be solved with the initial condition $\widetilde{q}(0)=1$, and Eq. (18) should be solved with the initial condition $\widetilde{S}(0)=\frac{S_{\mathrm{m} 0}}{A_{2} q_{\mathrm{m} 0}}<0$. Therefore, solutions of both equations depend on the sole parameter $\gamma$. Equations (17) and (18) are rigidly connected by balance Eq. (16).

Defining $x(\widetilde{t})=(\widetilde{q}(\widetilde{t}))^{1 / 3}$ and $\chi=|\gamma|^{1 / 3} \operatorname{sgn}(\gamma)$, the solution of Eq. (17) with the initial condition $x(0)=1$ is

$$
\begin{aligned}
2 \chi \tilde{t} & =\ln \left[\frac{(1-\chi)^{2}}{(x-\chi)^{2}} \frac{x^{2}+\chi x+\chi^{2}}{1+\chi+\chi^{2}}\right] \\
& +2 \sqrt{3}\left[\operatorname{atan} \frac{2 \sqrt{3} \chi(1-x)}{3 \chi^{2}+(2+\chi)(2 x+\chi)}\right] .
\end{aligned}
$$

The solution for the normalized supersaturation can be obtained from Eq. (20) and the balance Eq. (16)

$\widetilde{S}(\widetilde{t})=-\widetilde{q}(\widetilde{t})+\gamma=-x^{3}(\widetilde{t})+\chi^{3}$.

Figure 4 demonstrates time dependencies $\widetilde{S}(\widetilde{t})$ and $\widetilde{q}(\widetilde{t})$ calculated at different $\gamma$ at $S_{\mathrm{m} 0}>-10 \%$ (i.e., $\mathrm{RH}_{0}>90 \%$ ). As seen from Fig. 4 the analytical solution is quite close to the numerical one. The deviation increases with a decrease in parameter $\gamma$. At $\gamma=-0.5$, the error in the final $\mathrm{RH}$ is about $15 \%$. The initial $\mathrm{RH}_{0}$ in this case is about $90 \%$. $\mathrm{RH}_{0}$ is the relative humidity in the mixing volume $V$ after the first stage of mixing. As mentioned above, $\mathrm{RH}_{0}$ may be substantially higher than $\mathrm{RH}_{2}$ in the initially droplet-free volume. In situ measurements (Gerber et al., 2008) and remote measurements of aerosol humidification (Knight and Miller, 1998; Bar-Or et al., 2012) indicate the existence of zones of high RH along cloud edges. These observations and results of numerical simulations indicate that the analytical solution (Eqs. 20-21) is a universal one and applicable at any RH values in cloud surrounding.
The amplitude of the deviation of the analytical solution for supersaturation from the modeled result decreases with the decrease of $\gamma$. The cause of the deviation is neglecting term $(S+1)$ on the left-hand side of Eq. (8).

There are two types of solutions determined by parameter $\chi$ (or parameter $\gamma$ ), separated by value $\chi=0$ (Figs. 3 and 4). Condition $\chi=0$ corresponds to $\mu=\mu_{\mathrm{cr}}$ (see Pt1) and indicates complete evaporation of all the droplets and the relative humidity increasing up to $100 \%$. Condition $\chi>0$ corresponds to solutions $\widetilde{q}(\widetilde{t})$ with asymptotic behavior at $\widetilde{t} \rightarrow \infty: \widetilde{q} \rightarrow \gamma$ and $\widetilde{S} \rightarrow 0$, which means that droplets do not completely evaporate. Condition $\chi<0$ means that all the droplets completely evaporate.

At $\chi=0$, the analytical solution is

$x=\frac{3}{\widetilde{t}+3} ; \quad \widetilde{q}(\widetilde{t})=\left(\frac{3}{\widetilde{t}+3}\right)^{3} ; \quad \widetilde{S}(\widetilde{t})=-\left(\frac{3}{\widetilde{t}+3}\right)^{3}$.

Both LWC and supersaturation tend to zero at $\widetilde{t} \rightarrow \infty$.

At $\chi<0$, the duration of the evaporation is limited in time (Figs. 3, 4). Normalized evaporation time $t_{\mathrm{e}}$ depends on parameter $\chi$ only. This dependence can be obtained from Eq. (20) at $x=0$ :

$t_{\mathrm{e}}=\frac{1}{2 \chi} \ln \frac{(1-\chi)^{2}}{1+\chi+\chi^{2}}+\frac{\sqrt{3}}{\chi} \operatorname{atan} \frac{\sqrt{3}}{2 \chi+1}$.

Here the time is counted in the relaxation timescales. This fact indicates that the phase relaxation time is the timescale that should be used in the analysis of mixing. The choice of a timescale will be discussed in greater detail below.

At $\chi>-1 / 2$, one has to use the values $a \tan \left(\frac{\sqrt{3}}{2 \chi+1}\right)-\pi$ in Eq. (23) instead of $\operatorname{atan} \frac{\sqrt{3}}{2 \chi+1} \cdot t_{\mathrm{e}}$ is the time needed for supersaturation to reach its maximal value (Figs. 3a, 4a). This 

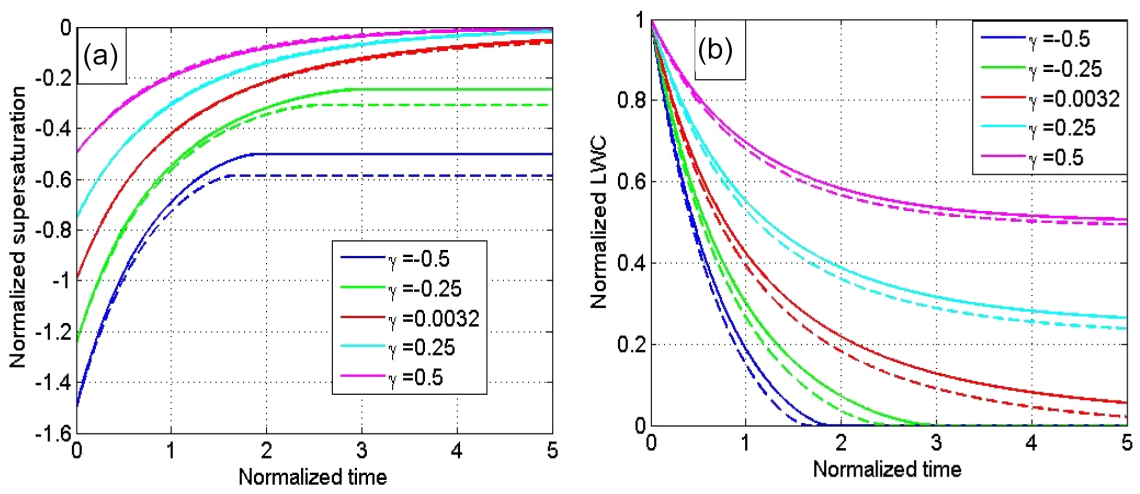

Figure 4. Universal dependencies $\widetilde{S}(\widetilde{t})$ (a) and $\widetilde{q}(\widetilde{t})$ (b), calculated at different values of parameter $\gamma$ using Eqs. (17)-(18) (solid lines) and using a parcel model (dashed lines).

value is calculated from Eqs. (16) and (6)

$$
\begin{aligned}
\widetilde{S}_{\max } & =\gamma=\chi^{3}=1+\frac{S_{\mathrm{m} 0}}{A_{2} q_{\mathrm{m} 0}} \approx 1+\frac{S_{2}}{A_{2} q_{1}} \frac{1-\mu}{\mu} \\
& =1+R \frac{1-\mu}{\mu}
\end{aligned}
$$

where $R=\frac{S_{2}}{A_{2} q_{1}}$ is a dimensionless parameter describing the ratio of $S_{2}$ in the initially dry air and the reserve of liquid water available for evaporation. (This parameter can be determined more precisely using Eq. 2 or 4 given in Pt1). This parameter can be referred to as a potential evaporation parameter (PEP). The PEP is proportional to the ratio of the amount of water vapor that should evaporate in order to saturate the initially droplet-free volume (that is determined by $S_{2}$ ) to the initial available liquid water $q_{1}$ in the cloud volume in case of equal initial volumes $V_{1}=V_{2}$.

The dependence $t_{\mathrm{e}}(\chi)$ is shown in Fig. 5. One can see that at large sub-saturation values $(\chi<-0.4)$ all the droplets evaporate within the span of a few relaxation times, and the analytical results agree well with the model (benchmark) results. At a high initial RH, droplet evaporation increases the humidity to nearly the saturation value, at which evaporation becomes extremely slow. In this case, simplified analytical formulas overestimate the evaporation time.

At $\chi>0$, droplets partially evaporate, $q$ reaches the minimal value and the thermodynamic equilibrium is reached when $S \rightarrow 0$. Minimal normalized equilibrium LWC can be found from Eqs. (21) and (6)

$\tilde{q}_{\min }=\gamma=\chi^{3}=1+R \frac{1-\mu}{\mu}$.

Eq. (25) is similar to Eqs. (4)-(5) (see Pt1).

Dependences of $\widetilde{S}_{\max }$ and $\widetilde{q}_{\min }$ on cloud air fraction, calculated at different initial conditions using Eqs. (24) and (25) are shown in Fig. 6. The diagram in Fig. 6b is actually a kind of widely used mixing diagram for homogeneous mixing, and Eq. (25) is a universal analytic equation for calcu-

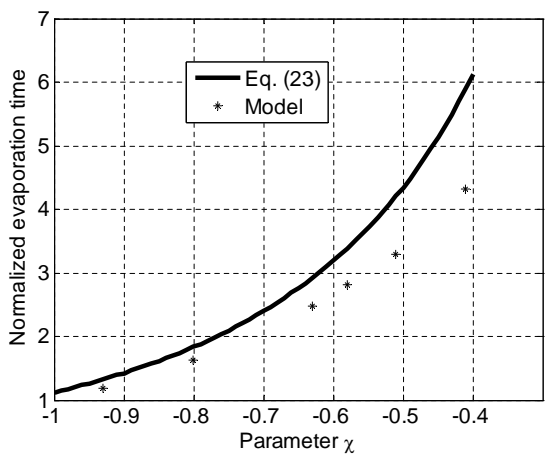

Figure 5. Dependence of the evaporation time on parameter $\chi$, $t_{\mathrm{e}}(\chi)$. Time is measured at the relaxation timescales. The values obtained using a parcel model are shown by asterisks.

lation of this diagram depending on the non-dimensional parameter $R$. Panel a shows that the higher the cloud fraction of air parcels involved in mixing, the lower the saturation deficit, i.e., the higher the final RH is. At small $R$, saturation is reached at a low $\mu$. Similar diagrams in physical units are shown in Pt1 in Fig. 4a, g.

The equations presented above allow to predict the results of homogeneous mixing both for partial and complete evaporation of droplets (Table 2). One can see that at low temperatures, the environment air volume becomes cloudy at relatively low values of liquid water content in the cloud volume even if RH of the environment volume is as low as $50 \%$. The reason is that the saturation ratio at low temperatures is low and only a small amount of liquid should be evaporated to make the initially dry volume saturated.

The diagram in Fig. $6 \mathrm{~b}$ allows to calculate the final liquid water content remaining in the entire volume after saturation reaches $100 \%$. This diagram also shows that for each value of $\mu$ there is a certain value of $R$ at which full evaporation takes place. The remaining $\widetilde{q}_{\text {min }}$ increases with decreasing $q_{1}$ and increasing $\mathrm{RH}_{2}$, as shown by the arrow. 
Table 2. Estimations of mixing results at different environmental conditions*.

\begin{tabular}{lrrrrrl}
\hline$T\left({ }^{\circ} \mathrm{C}\right)$ & $A_{2}$ & $q_{1}, \mathrm{~g} \mathrm{~kg}^{-1}$ & $\mathrm{RH}_{2} \%$ & $\mu$ (cloud fraction) & $R \frac{1-\mu}{\mu}$ & Result of mixing \\
\hline$T=0{ }^{\circ} \mathrm{C}$ & 400 & 1.0 & 95 & 0.5 & -0.125 & Cloudy \\
$T=0^{\circ} \mathrm{C}$ & 400 & 1.0 & 50 & 0.5 & -1.25 & Non-cloudy \\
$-10^{\circ} \mathrm{C}$ & 700 & 2.0 & 95 & 0.5 & -0.036 & Cloudy \\
$-10^{\circ} \mathrm{C}$ & 700 & 2.0 & 50 & 0.5 & -0.36 & Cloudy \\
$-20^{\circ} \mathrm{C}$ & 1500 & 2.0 & 95 & 0.5 & -0.017 & Cloudy \\
$-20^{\circ} \mathrm{C}$ & 1500 & 2.0 & 50 & 0.5 & -0.17 & Cloudy \\
$-20^{\circ} \mathrm{C}$ & 1500 & 2.0 & 95 & 0.1 & -0.153 & Cloudy \\
$-20^{\circ} \mathrm{C}$ & 1500 & 2.0 & 50 & 0.1 & -1.53 & Non-cloudy \\
\hline
\end{tabular}

* The values $A_{2}$ are estimated as proposed by Pinsky et al. (2013).
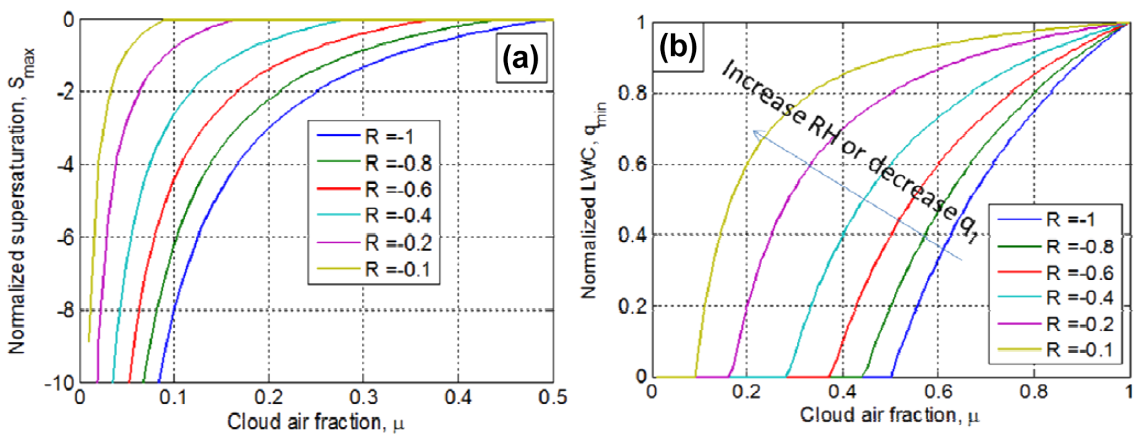

Figure 6. Dependencies of normalized equilibrium supersaturation $\widetilde{S}_{\max }$ (a) and normalized equilibrium LWC (which is equal to normalized equilibrium liquid water mixing ratio) $\widetilde{S}_{\max }$ (b) on cloudy air fraction at the final stage of homogeneous mixing. Curves of different colors correspond to different values of non-dimensional parameter $R=\frac{S_{2}}{A_{2} q_{1}}$.

The natural timescale of the evaporation and, therefore, homogeneous mixing was determined as $\tau_{\mathrm{m} 0}=$ $\left(B A_{2} N_{\mathrm{m} 0}^{2 / 3} q_{\mathrm{m} 0}^{1 / 3}\right)^{-1}$. This value is inversely proportional to $N_{\mathrm{m} 0} r_{\mathrm{m} 0}$ and actually coincides with the initial phase relaxation time $\tau_{\mathrm{pr}}$. The equality of the characteristic timescales of variations in supersaturation and in liquid water content directly follows from Eq. (15). Indeed, in case of $|S| \ll 1$ the equation can be rewritten as $S(t)=-A_{2} q(t)+C$ thus establishing a linear relationship between the supersaturation and the liquid water mixing ratio.

It should be emphasized that $\tau_{\mathrm{m} 0}$ is not the time of total droplet evaporation or the time of reaching saturation (when evaporation is over). The time of complete evaporation of all droplets can be substantially longer than phase relaxation time $\tau_{\mathrm{m} 0}$.

\section{Analysis of homogeneous droplet evaporation in case of a polydisperse DSD}

\subsection{DSD evolution in the course of droplet evaporation}

To analyze polydisperse DSD evolution during droplet evaporation at the second stage of homogeneous mixing, we use the same equations for diffusional growth (Eq. 9) and supersaturation (Eq. 8) as those used in case of a monodisperse DSD. The solution of Eq. (9) can be written in the form

$r^{2}(t)=r_{0}^{2}-Q(t)$,

where the non-negative function $Q(t) \geq 0$ is proportional to the supersaturation integral

$Q(t)=-\frac{2}{F} \int_{0}^{t} S\left(t^{\prime}\right) \mathrm{d} t^{\prime} \geq 0$.

This function characterizes a decrease in the square of droplet radii. At $t=0, Q(0)=0$. Let $f_{0}\left(r_{0}\right)$ be an initial DSD immediately following the first stage of homogeneous mixing. This distribution obeys the normalization condition

$N_{\mathrm{m} 0}=\int_{0}^{\infty} f_{0}\left(r_{0}\right) \mathrm{d} r_{0}$,

where $N_{\mathrm{m} 0}$ is the initial droplet number concentration after the first stage of mixing. Using the inverse transformation $r_{0}=\sqrt{r^{2}+Q(t)}$ alongside with condition $r \geq 0$ and the relation between the distribution functions $f(r, t)=f_{0}\left(r_{0}\right) \frac{\mathrm{d} r_{0}}{\mathrm{~d} r}$ 
Table 3. Parameters of the initial Gamma distributions.

\begin{tabular}{lrrrrr}
\hline DSD & $\begin{array}{r}N_{\mathrm{m} 0}, \\
\mathrm{~cm}^{-3}\end{array}$ & $\alpha$ & $\begin{array}{r}\beta, \\
\mu \mathrm{m}\end{array}$ & $\begin{array}{r}\text { Modal radius, } \\
\mu \mathrm{m}\end{array}$ & $\begin{array}{r}\text { LWC, } \\
\mathrm{g} \mathrm{m}^{-3}\end{array}$ \\
\hline Narrow & 264.2 & 101.0 & 0.1 & 10.0 & 0.587 \\
Wide & 71.0 & 4.3 & 3.1 & 10.0 & 0.587 \\
\hline
\end{tabular}

we get

$f(r, t)= \begin{cases}\frac{r}{\sqrt{r^{2}+Q(t)}} f_{0}\left(\sqrt{r^{2}+Q(t)}\right), & r \geq 0 \\ 0, & r<0\end{cases}$

Eq. (29) shows that the time changes in DSD and in its moments depend both on the initial DSD at $t=0, f_{0}\left(r_{0}\right)$ and on the time-dependent function $Q(t) \geq 0$.

To illustrate the DSD evolution using Eq. (29), we assume that the initial distribution immediately following the first stage of mixing can be represented by a Gamma distribution:

$f_{0}\left(r_{0}\right)=\frac{N_{\mathrm{m} 0}}{\Gamma(\alpha) \beta}\left(\frac{r_{0}}{\beta}\right)^{\alpha-1} \exp \left(-\frac{r_{0}}{\beta}\right)$,

where $N_{\mathrm{m} 0}$ is an intercept parameter, $\alpha$ is a shape parameter and $\beta$ is a slope parameter of distribution. Different sets of parameters allow approximations of both narrow and wide DSD. The parameters of the initial Gamma distribution used in this study are presented in Table 3 and are chosen so that the modal radii of DSD and the LWC would be the same for both distributions.

Combining Eqs. (29) and (30) yields an equation for DSD evolution as a function of $Q(t)$

$$
\begin{aligned}
& f(r, t)= \\
& \begin{cases}\frac{N_{\mathrm{m} 0}}{\Gamma(\alpha) \beta^{\alpha}}\left(r^{2}+Q\right)^{\frac{\alpha}{2}-1} r \exp \left(-\frac{\sqrt{r^{2}+Q}}{\beta}\right), & r \geq 0 \\
0, & r<0\end{cases}
\end{aligned}
$$

This DSD (Eq. 31) depends on four parameters, wherein parameter $Q(t)$ increases with time according to Eq. (27). Examples of evolutions of an initially narrow DSD and an initially wide DSD are shown in Fig. 7. All the calculations were performed using a parcel model (Korolev, 1995).

There is a significant difference between evolution of DSDs in cases of monodisperse and polydisperse DSDs. At a monodisperse DSD, droplet concentration remains unchanged until the final stage of evaporation when droplets become small and then all evaporate rapidly. At a polydisperse DSD the droplet concentration decreases simultaneously with the decrease in LWC. Evaporation of the narrow size distribution is consistent with the concept of homogeneous mixing (Fig. 7a). However homogeneous evaporation of droplets with a wide DSD may be mistakenly taken for inhomogeneous mixing.

\subsection{Evolution of DSD moments and related functions}

Equation (29) also allows evaluation of droplet concentration, DSD moments and related functions. Droplet concentration corresponds to the zero moment of DSD and can be expressed as

$$
\begin{aligned}
N(t) & =\int_{0}^{\infty} f(r, t) \mathrm{d} r=\int_{0}^{\infty} \frac{r}{\sqrt{r^{2}+Q(t)}} f_{0}\left(\sqrt{r^{2}+Q(t)}\right) \mathrm{d} r \\
& =\int_{\sqrt{Q(t)}}^{\infty} f_{0}\left(r_{0}\right) \mathrm{d} r_{0} .
\end{aligned}
$$

Since function $Q(t)$ monotonically increases with time, the right-hand integral in Eq. (32) decreases, indicating a decrease in droplet concentration with time. If the initial distribution of droplets is described by a Gamma distribution, the decrease of droplet concentration with the time is evaluated using Eqs. (30) and (32) as

$$
\begin{aligned}
N(t) & =\int_{\sqrt{Q(t)}}^{\infty} f_{0}\left(r_{0}, t\right) \mathrm{d} r_{0} \\
& =\int_{\sqrt{Q(t)}}^{\infty} \frac{N_{\mathrm{m} 0}}{\Gamma(\alpha) \beta}\left(\frac{r_{0}}{\beta}\right)^{\alpha-1} \exp \left(-\frac{r_{0}}{\beta}\right) \mathrm{d} r_{0} \\
& =\frac{N_{\mathrm{m} 0}}{\Gamma(\alpha)} \int_{\frac{\sqrt{Q(t)}}{\beta}}^{\infty} x^{\alpha-1} \exp (-x) \mathrm{d} x=N_{\mathrm{m} 0} \frac{\Gamma(\alpha, \eta)}{\Gamma(\alpha)},
\end{aligned}
$$

where

$\eta(t)=\frac{\sqrt{Q(t)}}{\beta}$

is a non-dimensional function of time and $\Gamma(\alpha, \eta(t))$ is an upper incomplete Gamma function (Korn and Korn, 2000). It follows from Eqs. (32) and (33) that $N(t) \leq N_{0}$. The dependencies of the normalized droplet concentration $\frac{N(t)}{N_{\mathrm{m} 0}}$ on time for an initially narrow DSD and an initially wide DSD are shown in Fig. 8.

Figure 8 shows that in case of an initially narrow DSD, droplet concentration does not change during the first $20 \mathrm{~s}$ when $\mathrm{RH}_{0}=91.6 \%$ because the DSD does not shift strongly enough toward smaller droplet radii. At lower initial supersaturations, droplet concentration decreases with time. This decrease may take place rapidly and may last several seconds only. The red line separates two different evaporation scenarios. The curves above the red line correspond to partial evaporation and reaching the saturation state, whereas the curves below the red line correspond to complete evaporation of droplets and the environment remains subsaturated.

At an initially wide DSD, droplet concentration decreases at any initial subsaturation value because the DSD contains 

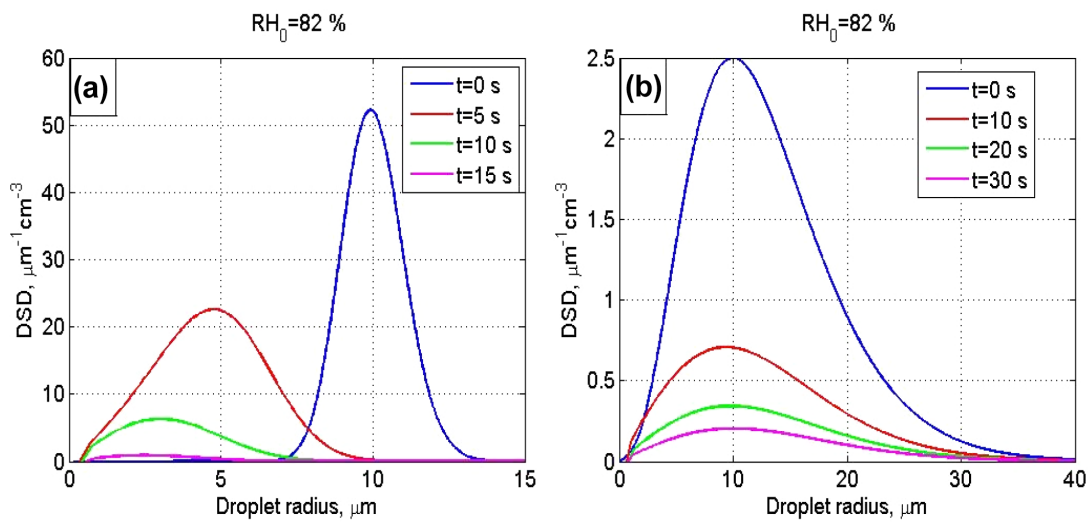

Figure 7. Time evolution of an initially narrow DSD (a) and an initially wide DSD (b). The initial calculation parameters are the same in both examples: $T_{0}=10^{\circ} \mathrm{C}, p=829 \mathrm{mb}, \mathrm{RH}_{0}=82 \%$ and $q_{\mathrm{w} 0}=0.587 \mathrm{~g} \mathrm{~m}^{-3}$. The parameters of the initial Gamma distributions are given in Table 3.
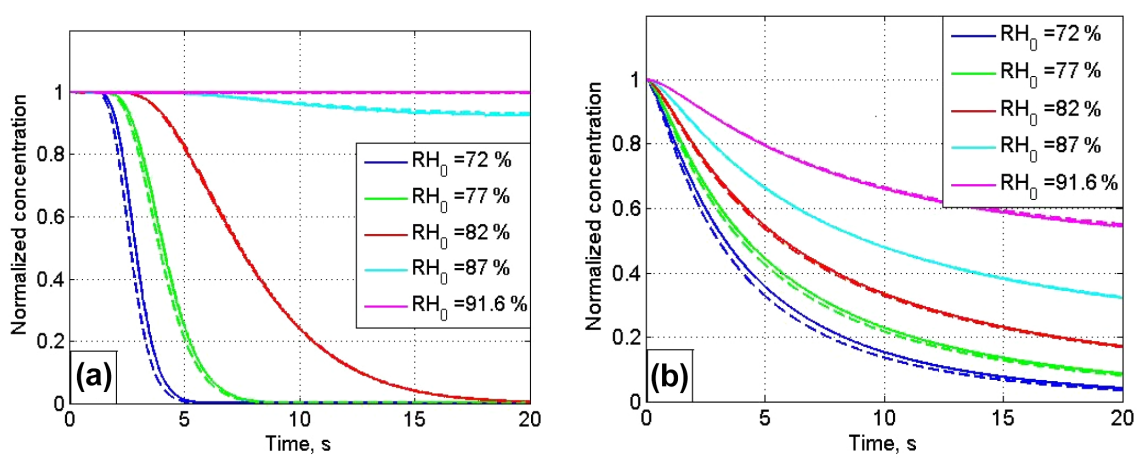

Figure 8. Time dependencies of normalized droplet concentration for an initially narrow DSD (a) and an initially wide DSD (b) at initially different values of $\mathrm{RH}_{0}$ in the resulting volume. The dependencies are calculated directly using a parcel model (solid lines) and using Eq. (33) (dashed lines). The thermodynamic parameters are the same as in Fig. 7. Parameters of the initial DSDs are given in Table 3.

small droplets that start evaporating regardless of the subsaturation value. In the particular example shown in Fig. 8, the droplet relaxation time is shorter in case of a wide DSD, so droplet concentration decreases slower than at a narrow DSD. Droplet concentration decreases substantially during a few tens of seconds, but does not reach zero due to a significant concentration of large droplets in the wide DSD. The equilibrium state is not reached within $20 \mathrm{~s}$.

Figure 8 also demonstrates an excellent agreement between the results of analytical calculations performed using Eq. (33) and results obtained using the parcel model.

A normalized moment of the $k$ th order is evaluated as

$$
\begin{aligned}
\overline{r^{k}(t)} & =\frac{1}{N(t)} \int_{0}^{\infty} r^{k} f(r, t) \mathrm{d} r \\
& =\frac{1}{N(t)} \int_{0}^{\infty} \frac{r^{k+1}}{\sqrt{r^{2}+Q(t)}} f_{0}\left(\sqrt{r^{2}+Q(t)}\right) \mathrm{d} r
\end{aligned}
$$

$$
\begin{gathered}
=\frac{1}{N(t)} \int_{\sqrt{Q(t)}}^{\infty}\left(r_{0}^{2}-Q(t)\right)^{k / 2} f_{0}\left(r_{0}\right) \mathrm{d} r_{0} \\
\overline{r^{k}(t)}=\frac{\int_{\sqrt{Q(t)}}^{\infty}\left(r_{0}^{2}-Q(t)\right)^{k / 2} f_{0}\left(r_{0}\right) \mathrm{d} r_{0}}{\int_{\sqrt{Q(t)}}^{\infty} f_{0}\left(r_{0}\right) \mathrm{d} r_{0}}
\end{gathered}
$$

In case when the initial distribution is given by the Gamma distribution (Eq. 30), Eq. (35) leads to the following equation

$$
\begin{aligned}
& \overline{r^{k}}(t)= \\
& \frac{\beta^{k}}{\Gamma(\alpha, \eta(t))} \int_{\eta(t)}^{\infty}\left(x^{2}-\eta^{2}(t)\right)^{k / 2}(x)^{\alpha-1} \exp (-x) \mathrm{d} x .
\end{aligned}
$$

The even moments can be represented using incomplete Gamma functions.

Figures 9 and 10 show the time dependencies of quantities typically used for characterizing the DSD shape, namely the 

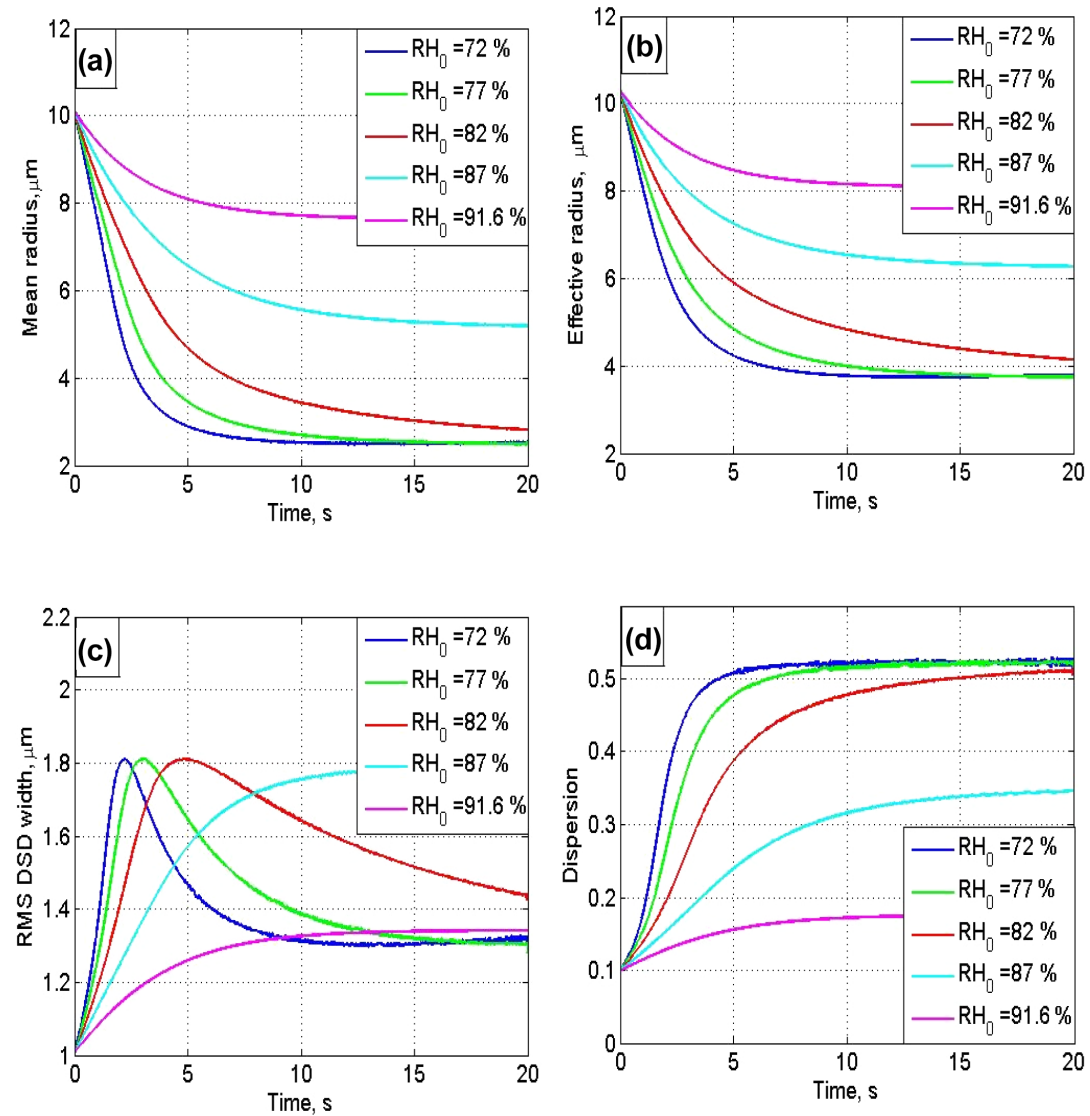

Figure 9. Dependencies of moment functions typically used for characterizing DSD shape at different values of the initial relative humidity $\mathrm{RH}_{0}$ in the resulting volume. The dependencies are calculated using a parcel model for an initially narrow DSD (Table 3 ). The thermodynamic parameters are the same as in Fig. 7.

mean radius $\bar{r}(t)$ (panel a), the effective radius $r_{\mathrm{eff}}(t)=\frac{\overline{r^{3}}(t)}{r^{2}(t)}$ (panel b), the RMS width of DSD $\sigma(t)=\sqrt{\overline{r^{2}}(t)-\bar{r}^{2}(t)}$ (panel c) and the dispersion coefficient $\delta(t)=\frac{\sigma(t)}{\bar{r}(t)}$ (panel d). The dependencies corresponding to an initially narrow DSD are shown in Fig. 9 and those corresponding to an initially wide DSD are shown in Fig. 10.

At an initially narrow DSD, the mean radius and the effective radius decrease with time in agreement with the concept of homogeneous mixing. Formation of plateaus in the mean radii, in the effective radii and in droplet dispersion over long time periods is caused by the existence of droplets in the tail of DSD distributions. While the concentration of such droplets is negligibly small, their evaporation takes a significant amount of time.

At an initially wide DSD (Fig. 7b), complete evaporation of the smallest droplets starts at the very beginning of the second stage of mixing. This evaporation leads to an increase in the effective radius and in the mean radius that changes non-monotonically. An increase in the value of the effective radius contradicts the concept of homogeneous mixing, according to which both the mean radii and the effective radii decrease in the course of mixing. This increase is explained by the fact that subsaturation at an initially wide DSD leads to a significant rapid decrease in the concentration of small droplets, while the changes in LWC whose value is determined by larger droplets, do not occur that quickly.

The opposite behaviors of the effective radii (as well as of the other characteristic droplet sizes) at a narrow DSD vs. a wide DSD, illustrated in Figs. 9 and 10, suggest the existence of a large number of DSDs with initial shapes at which the evaporation of droplets leads to a decrease in droplet concentration, but does not change the effective radius significantly. So the constancy of the effective radius at varying droplet concentrations does not allow to distinguish the mixing type with full confidence. Even at a narrow DSD, the decrease in the value of the effective radius does not exceed $20 \%$ at an initial saturation deficit of $8.4 \%$.

In any case the evolution of DSD and of their parameters is determined by the competition between two effects. First, this is the effect of partial droplet evaporation which shifts the DSD toward smaller sizes and leads to a decrease in the mean radii and in the effective radii, as well as widens the DSD. Second, this is the effect of complete evaporation of 

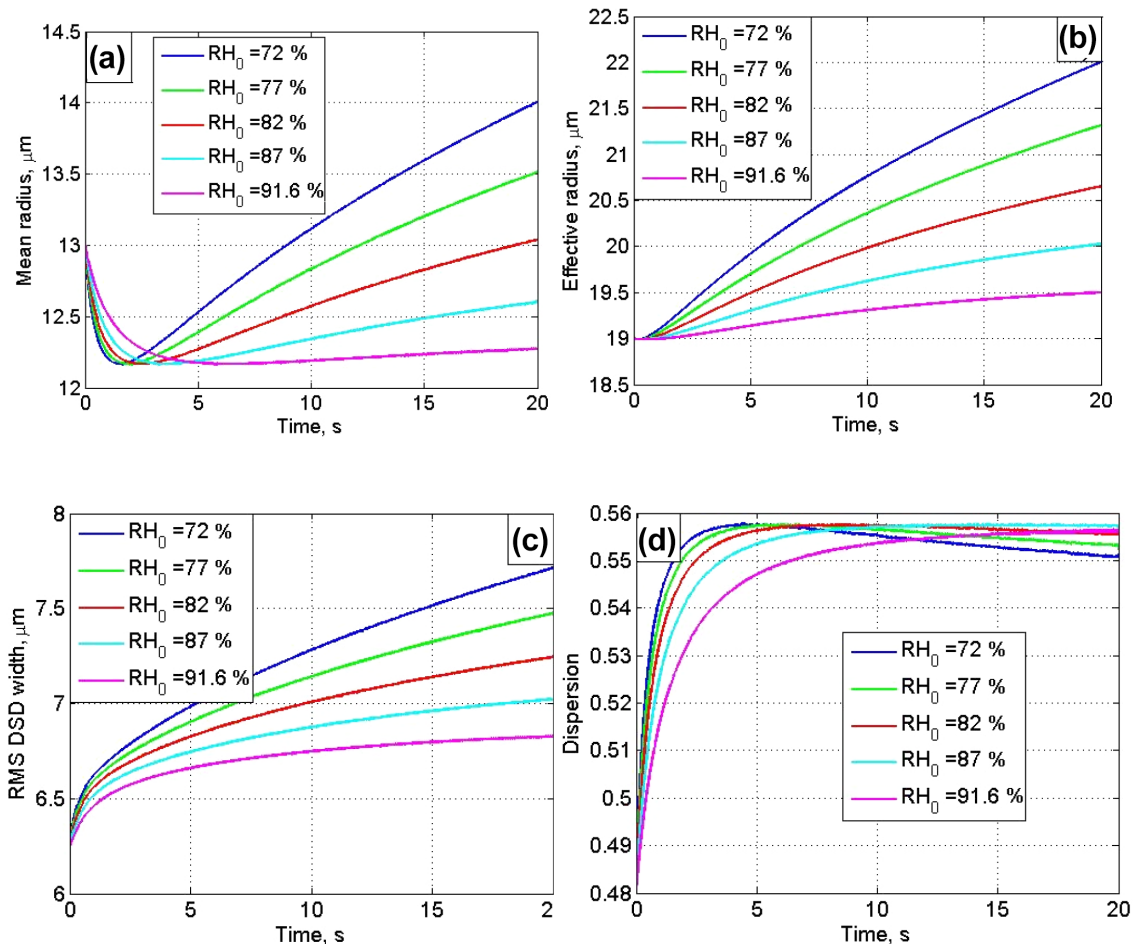

Figure 10. The same as in Fig. 9 but for an initially wide DSD.

the smallest droplets, which increases both the mean radii and the effective radii. The relative contribution of these two effects depends on the initial DSD width and the value of the mean radius. The best indicators of these two effects are the DSD width, $\sigma$ and the DSD dispersion $\frac{\sigma}{\bar{r}}$.

At an initially narrow DSD and an initially low RH, partial evaporation initially dominates and the DSD width increases due to the appearance of smaller droplets (Fig. 9c). Afterwards, when complete droplet evaporation becomes the dominant factor, the DSD shifts significantly to small sizes and the DSD width decreases. At an initially large RH, complete droplet evaporation is not efficient, and the DSD gets continuously wider. Since the mean radius decreases, the DSD dispersion tends to constant values at any initial RH. However, the largest DSD dispersion takes place at an initially low RH, when evaporation substantially decreases the mean droplet radius. As seen in Figs. 9d and 10d, the initial DSD dispersion at a narrow DSD is 0.1 , while the initial dispersion at a wide DSD is 0.5 .

Figure 10c and d show that at an initially wide DSD, homogeneous evaporation leads to an increase in both DSD width and DSD dispersion. The increase in the DSD width indicates that formation of the smallest droplets by partial evaporation is the main mechanism of the DSD shape evolution. The DSD dispersion increases with time and rapidly reaches quasi-stationary values of about 0.56 that are typical of real clouds.

\subsection{Evolution of LWC and of supersaturation}

Using Eq. (35), the time dependence of the liquid water mixing ratio is represented as

$$
\begin{aligned}
q(t) & =\frac{4 \pi \rho_{\mathrm{w}}}{3 \rho_{\mathrm{a}}} N(t) \overline{r^{3}(t)} \\
& =\frac{4 \pi \rho_{\mathrm{w}}}{3 \rho_{\mathrm{a}}} \int_{\sqrt{Q(t)}}^{\infty}\left(r_{0}^{2}-Q(t)\right)^{3 / 2} f_{0}\left(r_{0}\right) \mathrm{d} r_{0} .
\end{aligned}
$$

If the initial DSD is approximated by a Gamma distribution, $q(t)$ can be written as

$$
\begin{aligned}
q(t) & =\frac{4 \pi \rho_{\mathrm{w}}}{3 \rho_{\mathrm{a}}} \int_{\sqrt{Q(t)}}^{\infty}\left(r_{0}^{2}-Q(t)\right)^{3 / 2} \frac{N_{\mathrm{m} 0}}{\Gamma(\alpha) \beta}\left(\frac{r_{0}}{\beta}\right)^{\alpha-1} \\
& \exp \left(-\frac{r_{0}}{\beta}\right) \mathrm{d} r_{0}=\frac{4 \pi \rho_{\mathrm{w}}}{3 \rho_{\mathrm{a}}} \frac{N_{\mathrm{m} 0} \beta^{3}}{\Gamma(\alpha)} \\
& \int_{\eta(t)}^{\infty}\left(x^{2}-\eta^{2}(t)\right)^{3 / 2}(x)^{\alpha-1} \exp (-x) \mathrm{d} x .
\end{aligned}
$$

Since at $t=0, \eta=0$ and the initial liquid water mixing ratio is equal to $q_{\mathrm{m} 0}=\frac{4 \pi \rho_{\mathrm{w}}}{3 \rho_{\mathrm{a}}} N_{\mathrm{m} 0} \beta^{3} \frac{\Gamma(\alpha+3)}{\Gamma(\alpha)}$, hence the normalized 

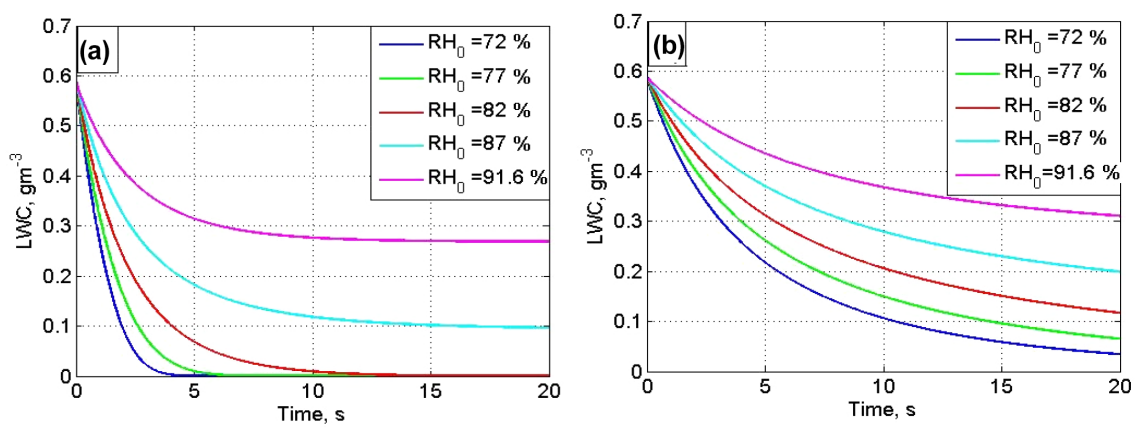

Figure 11. Time dependencies of LWC calculated using a parcel model at different values of $\mathrm{RH}_{0}$ in the resulting volume, for an initially narrow DSD (a) and an initially wide DSD (b). The thermodynamic parameters are the same as in Fig. 7. The parameters of the initial DSDs are given in Table 3.
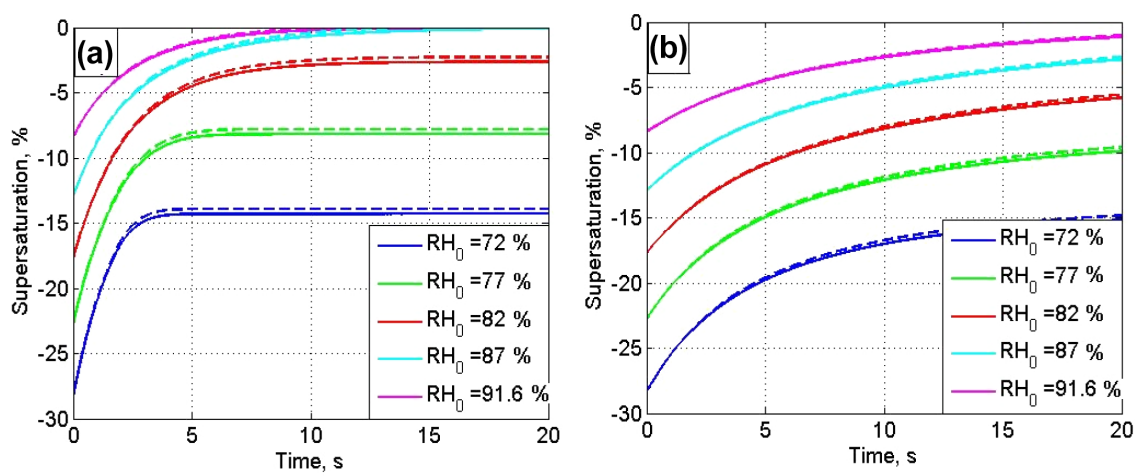

Figure 12. Time dependencies of supersaturation calculated at different values of the initial relative humidity $\mathrm{RH}_{0}$ in the resulting volume, using Eq. (8) (solid lines) and Eq. (40) (dashed lines), for an initially narrow DSD (a) and an initially wide DSD (b). The thermodynamic parameters are the same as in Fig. 7. The parameters of the initial DSDs are given in Table 3.

liquid water mixing ratio is calculated as

$$
\begin{gathered}
\frac{q(t)}{q_{\mathrm{m} 0}}=\frac{1}{\Gamma(\alpha+3)} \int_{\eta(t)}^{\infty}\left(x^{2}-\eta^{2}(t)\right)^{3 / 2} \\
(x)^{\alpha-1} \exp (-x) \mathrm{d} x .
\end{gathered}
$$

Figure 11 shows the time dependencies of LWC for different initial $\mathrm{RH}_{0}$ in the resulting volume (Fig. 11a: an initially narrow DSD; Fig. 11b: an initially wide DSD). One can see that at an initially narrow DSD the LWC rapidly decreases, either to zero (full evaporation) or, as in the monodisperse case, to an equilibrium value during a time period of the phase relaxation time (see Sect. 3 for comparison). At an initially wide DSD, the LWC decreases slowly and monotonically. In general, at an initially narrow DSDs the time dependencies, $q_{\mathrm{w}}(t)$ are quite close to those for monodisperse DSD (Fig. 3b). The higher the initial RH, the smaller the change in the LWC.

To calculate the time dependencies of supersaturation, one can use the full Eq. (8) or (15) written in the following form:

$S(t)=\left(S_{\mathrm{m} 0}+1\right) \exp \left\{-A_{2}\left[q(t)-q_{\mathrm{m} 0}\right]\right\}-1$.
In Eq. (39), $S_{\mathrm{m} 0}$ and $q_{\mathrm{m} 0}$ are the initial supersaturation and the liquid water mixing ratio, respectively, at $t=0$. The corresponding time dependencies are shown in Fig. 12. The analytical results of Eq. (40) are compared with the exact numerical solution obtained by a parcel model, showing a good agreement. The behavior of supersaturation at an initially narrow DSD is similar to that of a monodisperse DSD (Fig. 3a). As in Fig. 3a, the equilibrium non-zero values of subsaturation correspond to complete droplet evaporation. At initially wide DSD, the saturation deficit monotonically decreases as a consequence of the monotonic decrease in the LWC.

Figure 13 shows the dependencies of normalized LWC on normalized droplet concentrations calculated at different values of the initial relative humidity $\mathrm{RH}_{0}$ in the mixing volume (a: the initially narrow DSD and b: the initially wide DSD). Each point on the curves corresponds to a certain time. Since the dependencies are plotted in non-dimensional coordinates, the time instance $t=0$ corresponds to coordinates $(1,1)$. The numbers along the curves denote the points corresponding to time instance $t=20 \mathrm{~s}$. At lower $\mathrm{RH}_{0}$, the curves reach lower values of LWC and of droplet concen- 

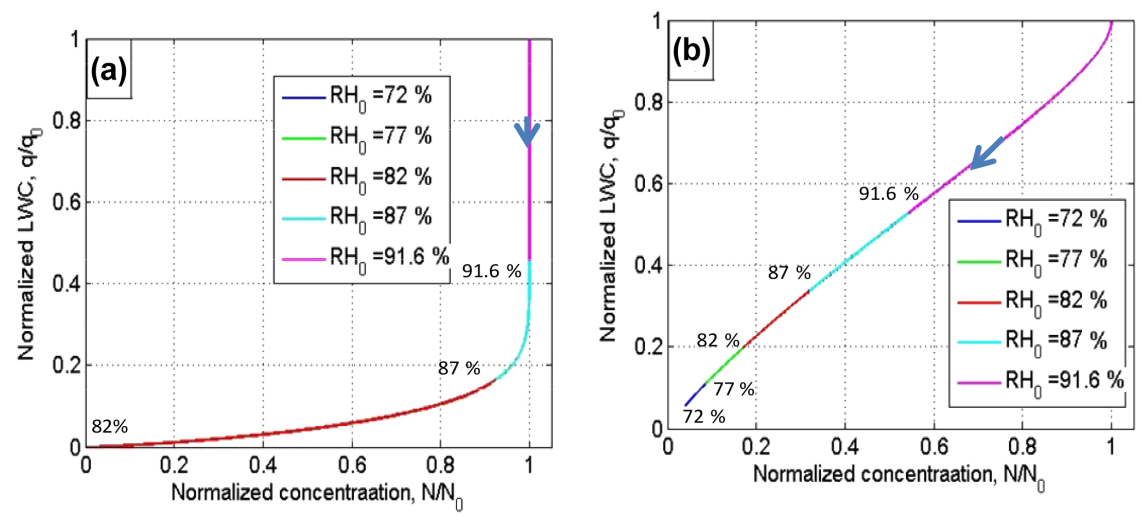

Figure 13. Dependencies of normalized LWC on the normalized number concentration of droplets calculated at different values of the initial relative humidity $\mathrm{RH}_{0}$ in the resulting volume, for an initially narrow DSD (a) and an initially wide DSD (b). The thermodynamic parameters are the same as in Fig. 7. The parameters of the initial DSDs are given in Table 3. Arrows denote the direction of increasing time.

tration. In case of an initially narrow DSD the curve can be divided into three sections. The first section corresponds to high $\mathrm{RH}_{0}\left(\mathrm{RH}_{0}>90 \%\right)$. Within this section, the droplet concentration does not decrease with decreasing LWC, which is in line with the conceptual scheme of homogeneous mixing (Fig. 1). This section corresponds to the straight horizontal line in Fig. 8a. At lower relative humidity, the droplet concentration begins to decrease with a decreasing LWC, in line with Fig. 8a. When the LWC reaches small values, the dependence of the normalized LWC on the normalized droplet concentration becomes close to linear. At an initially wide DSD (Fig. 13b), the dependence of the LWC on droplet concentration is close to linear at all the values of $\mathrm{RH}_{0}$. This linear dependence means that the mean volume radius varies only slightly during droplet evaporation.

It is noteworthy that all the curves plotted for different initial values of $\mathrm{RH}_{0}$ coincide. This coincidence can be explained by the fact that all the DSDs used depend on four parameters, namely, the three parameters of the initial Gamma distribution and on $Q(t)$ (Eq. 31). The parameters of Gamma distributions are identical for each panel in Fig. 13. Parameter $Q(t)$ monotonically increases with time. At different $\mathrm{RH}_{0}$, the values of $Q(t)$ reach the same values at different times. When the values of $Q(t)$ are the same, the DSDs and all the DSD moments are equal.

To sum up, at an initially narrow DSD and a comparatively large initial relative humidity $\mathrm{RH}_{0}>87 \%$ phenomenon demonstrates properties typically attributed to homogeneous mixing, when a decrease in both the LWC and the effective radius takes place at unchanged droplet concentration (Figs. 8a, 9b, 11a, 13a). In contrast, in the case of an initially wide DSD, the evolution of the DSD and its moments is close to that typically attributed to inhomogeneous mixing, when droplet evaporation leads to a decrease in LWC and in droplet concentration, while the effective radius remains unchanged. During the changes of a wide DSD, its shape remains similar to itself (Fig. 7b), which is also considered typical of inhomogeneous mixing.

\section{Discussion: application of the concept of homogeneous mixing in numerical modeling}

The procedure of mixing in any cloud model involves two steps. At the first step, the changes in microphysical values in each grid point are calculated using the turbulent flux divergences. In case mixing takes place between any two volumes represented by neighboring grid points, the mixing volume containing both grid points never becomes homogeneous (a fortiori, the microphysical values in these grid points do not become identical during one time step), so the spatial gradients of the microphysical variables remain between neighboring grid points. This step represents inhomogeneous mixing at resolving scales. Mixing algorithm in models does not operate with "final" equilibrium values, as assumed in the classical mixing concepts, but rather with current timedependent values. In contrast, the changes in the microphysical and thermodynamical variables in the volumes represented by one grid points are often considered uniform at each time step, and therefore, the modeled subgrid mixing is treated as homogeneous. Therefore, in most numerical models mixing is inhomogeneous at resolved scales, but homogeneous at subgrid scales.

The estimations in Table 1 indicate that mixing is homogeneous at scales lower than $\sim 0.5 \mathrm{~m}$. This means that to simulate homogeneous mixing explicitly, the grid spacing should be less than $0.5 \mathrm{~m}$. If such grid spacing is used, the separation between mixing types could be described explicitly. However, grid spacing in most models significantly exceeds this value. This fact brings up two questions: "What error is introduced when the spatial scale separating mixing types in models is much larger than $0.5 \mathrm{~m}$ ?" and "Why are spectral microphysics models with a resolution of $40-50 \mathrm{~m}$ able 
to reproduce observed DSD and their moments with high accuracy (Benmoshe et al., 2012; Khain et al., 2013, 2015; Magaritz-Ronen et al., 2014)?"

There are several factors that compensate errors in segregating mixing types in cloud models and allow using grid scale $L>L_{\mathrm{pr}}$ with little effect on DSD. The first factor is that mixing leads to formation of cloud zones characterized by a spatial correlation scale (radius of correlation) of temperature, humidity and droplet concentration of about 150$250 \mathrm{~m}$ (Magaritz-Ronen et al., 2014). Numerical experiments with Lagrangian-Eulerian model of Sc (Magaritz-Ronen et al., 2014) have shown that the results are not sensitive to the choice of parcel size, if this size is substantially smaller than the spatial radius of correlation. Therefore, the mixing type has a minor effect on the results of mixing at scales lower than the radius of correlation.

The second factor is that in-cloud mixing often takes place at conditions close to saturation. At such high humidity, homogeneous and inhomogeneous mixing yield practically the same results. The similarity of results for the two mixing types is due to the fact that mixing in clouds is not accompanied by an appreciable phase transition.

The third factor was pointed out by Hill et al. (2009), who explained that stratocumulus cloud evolution is insensitive to the type of sub-grid mixing since the rates of condensation and/or evaporation caused by the resolved dynamics are by 2 orders of magnitude greater than the condensation and/or evaporation rate caused by the sub-grid processes.

The fourth factor that permits us to treat sub-grid mixing as homogeneous near-cloud interfaces is that DSDs are polydisperse, which is opposite what is assumed in the conventional mixing considerations. In the present study it was shown that for a broad DSD, the changes of $r_{\text {eff }}$ remain small during mixing. So, a relatively small partial evaporation of droplets provide sufficient amount of water vapor for saturation of the volume. In this case homogeneous mixing becomes indistinguishable from inhomogeneous. The saturation of the volume may be facilitated by entrainment of water vapor from neighboring cloud volumes.

\section{Conclusions}

The present study is focused on the dynamics of DSD transformation during the evaporation stage of homogeneous mixing. The results can be summarized as follows.

1. Analytical equations describing time evolution of normalized supersaturation and normalized LWC are obtained. It is found that these time dependences are universal functions of a sole non-dimensional parameter $\gamma=1+\frac{S_{\mathrm{m} 0}}{A_{2} q_{\mathrm{m} 0}}$. In particular, the dependences of normalized LWC at the final stage on the cloud air fraction, used for plotting the universal mixing diagrams, are obtained analytically. These diagrams also depend on a sole non-dimensional parameter $R=\frac{S_{2}}{A_{2} q_{1}}<0$, which is proportional to supersaturation in dry volume and inversely proportional to liquid water mixing ratio in a cloud volume. This parameter is uniquely related with parameter $\gamma$ by Eq. (24). It is shown that in many cases the major changes in the LWC take place during the time period of the order of the phase relaxation time $\tau_{\mathrm{pr}}$. The equilibrium state can be reached after several $\tau_{\text {pr periods. }}$

2. It is shown that the phase relaxation time is a natural timescale of mixing process. This is clearly seen from the universal renormalized evaporation equations, in which the phase relaxation time plays the role of a time unit. In some studies (e.g., Baker and Latham, 1979; Burnet and Brenguier, 2007; Andejchuk et al., 2009) evaporation time for an individual droplet under given sub-saturation is considered as a characteristic time of mixing. The present study shows that only the phase relaxation time should be used as the characteristic timescale of mixing since we have to consider the behavior of a large amount of droplets. Supersaturation (or sub-saturation) is not a parameter that determines the phase relaxation time. Thus, the utilization of the evaporation time of individual droplet (at unchanged supersaturation) as the characteristic timescale of mixing is physically ungrounded. A strict relationship between the changes in supersaturation and in the liquid water mixing ratio makes it impossible to consider the changes in an individual droplet size and in supersaturation independently.

3. An important outcome of this study is demonstration of a significant difference in the evaporative behavior between narrow DSD and wide DSD. It is shown that homogeneous evaporation of a wide DSD is accompanied by reduction in LWC and in droplet concentration due to total evaporation of small droplets. Such changes of LWC and droplet concentration are qualitatively different from those in the classic concept of homogeneous mixing. As a result, homogeneous mixing may be erroneously interpreted as inhomogeneous one.

4. It is shown that the evolution of DSDs and their moments in case of polydisperse DSDs, can qualitatively differ from that predicted by homogeneous mixing concept. Evaporation of a comparatively wide DSD may even lead to an increase in the effective radii and DSD high moments. This feature is typically attributed to inhomogeneous mixing.

Note that the role of DSD polydispersity in the mixing process is different from that in diffusion droplet growth in ascending parcels. In an ascending adiabatic parcel the supersaturation tends to zero with height, and the DSD width decreases with height as well. In this case, it is possible to reproduce the height dependencies and the time dependencies of supersaturation and of LWC using an "equivalent" 
monodisperse DSD with the same droplet concentration as polydisperse DSDs (Pinsky et al., 2014). As regards to mixing with a polydisperse DSD, it cannot be reproduced using a monodisperse DSD, with a possible exception in case of an initially extremely narrow DSD.

\section{Data availability}

The model codes are available upon request. 


\section{Appendix A: List of symbols}

Table A1. List of symbols.

\begin{tabular}{|c|c|c|}
\hline Symbol & Description & Units \\
\hline$A_{2}$ & $\frac{1}{q_{\mathrm{v}}}+\frac{L^{2}}{c_{p} R_{\mathrm{v}} T^{2}}$, coefficient & nd \\
\hline$B$ & $\frac{3}{F}\left(\frac{4 \pi \rho_{\mathrm{w}}}{3 \rho_{\mathrm{a}}}\right)^{2 / 3}$, coefficient & $\mathrm{m}^{2} \mathrm{~s}^{-1}$ \\
\hline C & constant of integration & nd \\
\hline$c_{p}$ & specific heat capacity of moist air at constant pressure & $\mathrm{J} \mathrm{kg}^{-1} \mathrm{~K}^{-1}$ \\
\hline$D$ & coefficient of water vapor diffusion in the air & $\mathrm{m}^{2} \mathrm{~s}^{-1}$ \\
\hline$e$ & water vapor pressure & $\mathrm{Nm}^{-2}$ \\
\hline$e_{s}$ & saturation vapor pressure above a flat water surface & $\mathrm{Nm}^{-2}$ \\
\hline$F$ & $F=\frac{\rho_{\mathrm{w}} L^{2}}{k_{\mathrm{a}} R_{\mathrm{v}} T^{2}}+\frac{\rho_{\mathrm{w}} R_{\mathrm{v}} T}{e_{s}(T) D}$, coefficient & $\mathrm{m}^{-2} \mathrm{~s}$ \\
\hline$f(r, t)$ & droplet size distribution & $\mathrm{m}^{-4}$ \\
\hline$f_{0}\left(r_{0}\right)$ & droplet size distribution after the first stage of mixing & $\mathrm{m}^{-4}$ \\
\hline$k_{\mathrm{a}}$ & coefficient of air heat conductivity & $\mathrm{J} \mathrm{m}^{-1} \mathrm{~s}^{-1} \mathrm{~K}^{-1}$ \\
\hline$L_{\text {mix }}$ & characteristic spatial scale of mixing & $\mathrm{m}$ \\
\hline$L_{\mathrm{pr}}$ & spatial scale of phase relaxation & $\mathrm{m}$ \\
\hline$L$ & latent heat for liquid water & $\mathrm{J} \mathrm{kg}^{-1}$ \\
\hline$N$ & droplet concentration & $\mathrm{m}^{-3}$ \\
\hline$N_{1}$ & droplet concentration in a cloud volume & $\mathrm{m}^{-3}$ \\
\hline$N_{\mathrm{m} 0}$ & droplet concentration after the first stage of mixing & $m^{-3}$ \\
\hline$p$ & pressure of moist air & $\mathrm{Nm}^{-2}$ \\
\hline$q$ & liquid water mixing ratio & $\mathrm{kg} \mathrm{kg}^{-1}$ \\
\hline$q_{1}$ & liquid water mixing ratio in a cloudy volume & $\mathrm{kg} \mathrm{kg}^{-1}$ \\
\hline$q_{\mathrm{m} 0}$ & liquid water mixing ratio after the first stage of mixing & $\mathrm{kg} \mathrm{kg}^{-1}$ \\
\hline$q_{\mathrm{w}}$ & liquid water content (LWC) & $\mathrm{g} \mathrm{m}^{-3}$ \\
\hline$q_{\mathrm{w} 0}$ & LWC after the first stage of mixing & $\mathrm{g} \mathrm{m}^{-3}$ \\
\hline$q_{\mathrm{v}}$ & water vapor mixing ratio & $\mathrm{kg} \mathrm{kg}^{-1}$ \\
\hline$\widetilde{q}$ & normalized liquid water mixing ratio equal to normalized LWC & nd \\
\hline$\widetilde{q}_{\min }$ & normalized equilibrium liquid water mixing ratio equal to normalized equilibrium LWC & nd \\
\hline$Q$ & change of square of droplet radius & $\mathrm{m}^{2}$ \\
\hline$r$ & droplet radius & $\mathrm{m}$ \\
\hline$R$ & $\frac{S_{2}}{A_{2} g_{1}}$, potential evaporation parameter $(\mathrm{PEP})$ & nd \\
\hline$R_{\mathrm{a}}$ & specific gas constant of moist air & $\mathrm{J} \mathrm{kg}^{-1} \mathrm{~K}^{-1}$ \\
\hline$R_{\mathrm{V}}$ & specific gas constant of water vapor & $\mathrm{J} \mathrm{kg}^{-1} \mathrm{~K}^{-1}$ \\
\hline $\mathrm{RH}_{0}$ & $1+S_{\mathrm{m} 0}$, relative humidity after the first stage of mixing & nd \\
\hline$S$ & $e / e_{\mathrm{w}}-1$, supersaturation over water & nd \\
\hline$S_{\mathrm{m} 0}$ & supersaturation after the first stage of mixing & nd \\
\hline$\underline{S}_{2}$ & supersaturation in a dry volume & nd \\
\hline$\widetilde{S}_{\max }$ & maximal normalized supersaturation & nd \\
\hline$\tilde{S}$ & normalized supersaturation & nd \\
\hline$T$ & temperature & K \\
\hline$T_{1}$ & temperature in a cloud volume & $\mathrm{K}$ \\
\hline$T_{2}$ & temperature in a dry volume & K \\
\hline$t_{\mathrm{e}}$ & normalized evaporation time & nd \\
\hline$t$ & time & $\mathrm{s}$ \\
\hline$\widetilde{t}$ & non-dimensional time & nd \\
\hline$x(\widetilde{t})$ & non-dimensional variable & nd \\
\hline$\alpha$ & parameter of the Gamma distribution & nd \\
\hline$\beta$ & parameter of the Gamma distribution & $\mathrm{m}^{-1}$ \\
\hline$\chi$ & $\gamma^{1 / 3}$, non-dimensional parameter & nd \\
\hline$\varepsilon$ & turbulent dissipation rate & $\mathrm{m}^{2} \mathrm{~s}^{-3}$ \\
\hline$\gamma$ & $1+\frac{S_{\mathrm{m} 0}}{A_{2} q_{\mathrm{m} 0}}$, non-dimensional parameter & nd \\
\hline$\delta q_{\mathrm{m}}$ & mixing ratio of liquid water required to saturate $1 \mathrm{~kg}$ of the cloud (or cloudy?) volume after instant mixing & nd \\
\hline$\mu$ & mass fraction of cloud air & nd \\
\hline$\rho_{\mathrm{a}}$ & air density & $\mathrm{kg} \mathrm{m}^{-3}$ \\
\hline$\rho_{\mathrm{W}}$ & density of liquid water & $\mathrm{kg} \mathrm{m}^{-3}$ \\
\hline$\tau_{\mathrm{pr}}$ & phase relaxation time & $\mathrm{s}$ \\
\hline$\tau_{\text {mix }}$ & characteristic time of mixing & s \\
\hline$\tau_{\mathrm{m} 0}$ & timescale & $\mathrm{s}$ \\
\hline
\end{tabular}

"nd" denotes non-dimensional. 
Appendix B: Derivation of closed equations for supersaturation and for liquid water mixing ratio in the monodisperse DSD case

Let us consider motionless well-mixed adiabatic air volume having an initial supersaturation $S_{\mathrm{m} 0}<0$ and an initial liquid water mixing ratio $q_{\mathrm{m} 0}$.

1. Substitution of the formula of the liquid water mixing ratio $q=\frac{4 \pi \rho_{\mathrm{w}}}{3 \rho_{\mathrm{a}}} N r^{3}$ into the equation for droplet radius evolution

$r \frac{\mathrm{d} r}{\mathrm{~d} t}=\frac{S}{F}$

leads to the equation for the decrease of $q$ with the time

$\frac{\mathrm{d} q}{\mathrm{~d} t}=\frac{3}{F}\left(\frac{4 \pi \rho_{\mathrm{w}}}{3 \rho_{\mathrm{a}}}\right)^{2 / 3} N^{2 / 3} S q^{1 / 3}=B N^{2 / 3} S q^{1 / 3}$,

where

$B=\frac{3}{F}\left(\frac{4 \pi \rho_{\mathrm{w}}}{3 \rho_{\mathrm{a}}}\right)^{2 / 3}=$ const.

2. Equation for supersaturation is written as (Korolev and Mazin, 2003)

$\frac{1}{S+1} \frac{\mathrm{d} S}{\mathrm{~d} t}=-A_{2} \frac{\mathrm{d} q}{\mathrm{~d} t}$.

Integration of this equation under the assumption that $A_{2}=$ const leads to the equation

$\ln [S(t)+1]=-A_{2} q(t)+C$,

where $C$ is determined by initial conditions at $t=0$

$C=\ln \left(S_{\mathrm{m} 0}+1\right)+A_{2} q_{\mathrm{m} 0}$.

Using Eqs. (B5) and (B6) one obtains the equation with respect to $S(t)$

$S(t)=\left(S_{\mathrm{m} 0}+1\right) \exp \left\{-A_{2}\left[q(t)-q_{\mathrm{m} 0}\right]\right\}-1$.

3. Mutual substitution of Eqs. (B2) and (B7) leads to the closed differential equations for $q(t)$ and $S(t)$

$$
\begin{gathered}
\frac{\mathrm{d} q}{\mathrm{~d} t}=B N^{2 / 3}\left[\left(S_{\mathrm{m} 0}+1\right) \exp \left\{-A_{2}\left(q-q_{\mathrm{m} 0}\right)\right\}\right. \\
-1] q^{1 / 3} \\
\frac{1}{S+1} \frac{\mathrm{d} S}{\mathrm{~d} t}=-A_{2} B N^{2 / 3} S\left(q_{\mathrm{m} 0}\right. \\
\left.-\frac{1}{A_{2}} \ln \frac{S+1}{S_{\mathrm{m} 0}+1}\right)^{1 / 3} .
\end{gathered}
$$

Eqs. (B8) and (B9) should be solved with initial conditions $q(0)=q_{\mathrm{m} 0}$ and $S(0)=S_{\mathrm{m} 0}$ respectively.
4. In case $\left|S_{\mathrm{m} 0}\right| \ll 1$, supersaturation is close to zero all the time $|S(t)| \ll 1$ and Eqs. (B7)-(B9) can be simplified as follows

$$
\begin{aligned}
& S(t)=S_{\mathrm{m} 0}-A_{2}\left[q(t)-q_{\mathrm{m} 0}\right] \\
& \frac{\mathrm{d} q}{\mathrm{~d} t}=-B N^{2 / 3}\left(A_{2} q^{4 / 3}-\left(A_{2} q_{\mathrm{m} 0}+S_{\mathrm{m} 0}\right) q^{1 / 3}\right) \\
& \frac{\mathrm{d} S}{\mathrm{~d} t}=-B\left(A_{2} N\right)^{2 / 3}\left(A_{2} q_{\mathrm{m} 0}+S_{\mathrm{m} 0}-S\right)^{1 / 3} S .
\end{aligned}
$$

5. Then one can obtain Eqs. (B10)-(B12) in a nondimensional form. Let us define timescale $\tau_{\mathrm{m} 0}=$ $\left(B A_{2} N^{2 / 3} q_{\mathrm{m} 0}^{1 / 3}\right)^{-1}$, normalized liquid water mixing ratio $\widetilde{q}=\frac{q}{q_{\mathrm{m} 0}}$, normalized supersaturation $\widetilde{S}=\frac{S}{A_{2} q_{\mathrm{m} 0}}$, and non-dimensional time $\tilde{t}=t / \tau_{\mathrm{m} 0}=B A_{2} N^{2 / 3} q_{\mathrm{m} 0}^{1 / 3} t$. The Eqs. (B10)-(B12) can be rewritten in a non-dimensional form as

$\widetilde{S}(\widetilde{t})=-\widetilde{q}(\widetilde{t})+\gamma$

$\frac{\mathrm{d} \widetilde{q}}{\mathrm{~d} \widetilde{t}}=\widetilde{q}^{1 / 3}(\gamma-\widetilde{q})$

$\frac{\mathrm{d} \widetilde{S}}{\mathrm{~d} \widetilde{t}}=-(\gamma-\widetilde{S})^{1 / 3} \widetilde{S}$

where non-dimensional parameter $\gamma=1+\frac{S_{\mathrm{m} 0}}{A_{2} q_{\mathrm{m} 0}}$ depends on initial supersaturation $S_{\mathrm{m} 0}$ and initial liquid water mixing ratio $q_{\mathrm{m} 0}$. Equation (B14) should be solved with initial condition $\widetilde{q}(0)=1$ and Eq. (B15) should be solved with initial condition $\widetilde{S}(0)=\frac{S_{\mathrm{m} 0}}{A_{2} q_{\mathrm{m} 0}}<$ 0 . Note that Eqs. (B14) and (B15) are rigidly connected by Eq. (B13). 
Acknowledgements. This research was supported by the Israel Science Foundation (grant 1393/14), the Office of Science (BER), the US Department of Energy Award DE-SC0006788 and the Binational US-Israel Science foundation (grant 2010446). A. Korolev's participation was supported by Environment Canada.

Edited by: T. Garrett

Reviewed by: two anonymous referees

\section{References}

Andejchuk, M., Grabowski, W. W., Malinowski, S. P., and Smolarkiewicz, P. K.: Numerical simulation of cloud-clear air interfacial mixing: homogeneous vs. inhomogeneous mixing, J. Atmos. Sci., 66, 2493-2500, 2009.

Baker, M. and Latham, J.: The evolution of droplet spectra and the rate of production of embyonic raindrops in small cumulus clouds, J. Atmos. Sci., 36, 1612-1615, 1979.

Baker, M., Corbin, R. G., and Latham, J.: The influence of entrainment on the evolution of cloud drop spectra: I. A model of inhomogeneous mixing, Q. J. Roy. Meteor. Soc., 106, 581-598, 1980.

Baker, M. B. and Latham, J.: A diffusive model of the turbulent mixing of dry and cloudy air, Quart. J. Roy. Meteor. Soc., 108, 871-898, 1982

Bar-Or, R. Z., Koren, I., Altaratz, O., and Fredj, E.: Radiative properties of humidified aerosol in cloudy environment, Atmos. Res., 118, 280-294, 2012.

Benmoshe, N., Pinsky, M., Pokrovsky, A., and Khain, A.: Turbulent effects on microstructure and precipitation of deep convective clouds as seen from simulations with a 2-D spectral microphysics cloud model, J. Geophys. Res., 117, D06220, doi:10.1029/2011JD016603, 2012.

Blyth, A. M., Choularton, T. W., Fullarton, G., Latham, J., Mill, C. S., Smith, M. H., and Stromberg, I. M.: The Influence of entrainment on the evolution of cloud droplet spectra. 2. Field experiments 5 at Great Dun Fell, Q. J. Roy. Meteor. Soc., 106, 821-840, 1980.

Burnet, F. and Brenguier, J.-L.: Observational study of the entrainment-mixing process in warm convective clouds, J. Atmos. Sci., 64, 1995-2011, 2007.

Devenish, B. J., Bartello, P., Brenguier, J.-L., Collins, L. R., Grabowski, W. W., Ijzermans, R. H. A., Malinovski, S. P., Reeks, M. W., Vassilicos, J. C., Wang, L.-P., and Warhaft, Z.: Droplet growth in warm turbulent clouds, Q. J. Roy. Meteor. Soc., 138, 1401-1429, 2012.

Gerber, H., Frick, G., Jensen, J. B., and Hudson, J. G.: Entrainment, mixing, and microphysics in trade-wind cumulus, J. Meteorol. Soc. Jpn., 86A, 87-106, 2008.

Hill, A. A., Feingold, G., and Jiang, H.: The influence of entrainment and mixing assumption on aerosol-cloud interactions in marine stratocumulus, J. Atmos. Sci., 66, 1450-1464, 2009.

Jeffery, C. A.: Inhomogeneous cloud evaporation, invariance, and Damköhler number, J. Geophys. Res., 112, D24S21, doi:10.1029/2007JD008789, 2007.
Khain, A., Thara, V., Prabha, N. B., Pandithurai, G., and Ovchinnikov, M.: The mechanism of first raindrops formation in deep convective clouds, J. Geophys. Res.-Atmos., 118, 9123-9140, 2013.

Khain, A. P., Beheng, K. D., Heymsfield, A., Korolev, A., Krichak, S. O., Levin, Z., Pinsky, M., Phillips, V., Prabhakaran, T., Teller, A., van den Heever, S. C., and Yano, J.-I.: Representation of microphysical processes in cloud-resolving models: spectral (bin) microphysics vs. bulk parameterization, Rev. Geophys., 53, 247322, 2015.

Knight, C. A. and Miller, L. J.: Early radar echoes from small, warm cumulus: Bragg and hydrometeor scattering, J. Atmos. Sci., 55, 2974-2992, 1998.

Korn, G. A. and Korn, T. M.: Mathematical handbook for scientists and engineers: Definitions, theorems, and formulas for reference and review, Courier Corporation - Mathematics, 1130 pp., 2000.

Korolev, A., Khain, A., Pinsky, M., and French, J.: Theoretical study of mixing in liquid clouds - Part 1: Classical concepts, Atmos. Chem. Phys., 16, 9235-9254, doi:10.5194/acp-16-92352016, 2016.

Korolev, A. V.: The influence of suresaturation fluctuations on droplet size spectra formation, J. Atmos. Sci., 52, 3620-3634, 1995.

Korolev, A. V. and Isaac, G. A.: Drop growth due to high supersaturation caused by isobaric mixing, J. Atmos. Sci., 57, 1675-1685, 2000.

Korolev, A. V. and Isaac, G. A.: Phase transformation of mixedphase clouds, Q. J. Roy. Meteorol. Soc., 129, 19-38, 2003.

Korolev, A. V. and Mazin, I. P.: Supersaturation of water vapor in clouds, J. Atmos. Sci., 60, 2957-2974, 2003.

Latham, J. and Reed, R. L.: Laboratory studies of effects of mixing on evolution of cloud droplet spectra, Q. J. Roy. Meteor. Soc., 103, 297-306, 1977.

Lehmann, K., Siebert, H., and Shaw, R. A.: Homogeneous and inhomogeneous mixing in cumulus clouds: Dependence on local turbulence structure, J. Atmos. Sci., 66, 3641-3659, 2009.

Mazin, I. P.: Effect of phase transition on formation of temperature and humidity stratification in clouds, Proc. Int. Conf. on Cloud Physics, Toronto, Ontario, Canada, Amer. Meteor. Soc., 132-137, 1968.

Magaritz-Ronen, L., Pinsky, M., and Khain, A.: Effects of turbulent mixing on the structure and macroscopic properties of stratocumulus clouds demonstrated by a Lagrangian trajectory model, J. Atmos. Sci., 71, 1843-1862, 2014.

Monin, A. S. and Yaglom, A. M.: Statistical Fluid Mechanics: Mechanics of Turbulence, Vol. 2, MIT Press, 911 pp., 1975.

Pinsky, M., Mazin, I. P., Korolev, A., and Khain, A.: Supersaturation and diffusional drop growth in liquid clouds, J. Atmos. Sci., 70, 2778-2793, 2013.

Pinsky, M., Mazin, I. P., Korolev, A., and Khain, A.: Supersaturation and diffusional droplet growth in liquid clouds: Polydisperse spectra, J. Geophys. Res.-Atmos., 119, 12872-12887, 2014.

Pinsky, M., Khain, A., and Korolev, A.: Theoretical analysis of mixing in liquid clouds - Part 3: Inhomogeneous mixing, Atmos. Chem. Phys., 16, 9273-9297, doi:10.5194/acp-16-92732016, 2016.

Pruppacher, H. R. and Klett, J. D.: Microphysics of clouds and precipitation, 2nd Edn., Oxford Press, 914 pp., 1997. 